TRANSACTIONS OF THE

AMERICAN MATHEMATICAL SOCIETY

Volume 353, Number 4, Pages 1587-1634

S 0002-9947(00)02715-X

Article electronically published on November 21, 2000

\title{
SYMMETRY-BREAKING BIFURCATION OF ANALYTIC SOLUTIONS TO FREE BOUNDARY PROBLEMS: AN APPLICATION TO A MODEL OF TUMOR GROWTH
}

\author{
AVNER FRIEDMAN AND FERNANDO REITICH
}

\begin{abstract}
In this paper we develop a general technique for establishing analyticity of solutions of partial differential equations which depend on a parameter $\varepsilon$. The technique is worked out primarily for a free boundary problem describing a model of a stationary tumor. We prove the existence of infinitely many branches of symmetry-breaking solutions which bifurcate from any given radially symmetric steady state; these asymmetric solutions are analytic jointly in the spatial variables and in $\varepsilon$.
\end{abstract}

\section{The MOdel AND MAIN RESUlt}

In this paper we present a general technique for establishing analyticity of solutions of systems of partial differential equations which depend analytically on a parameter $\varepsilon$. The method works not only for boundary value problems but also for free boundary problems. In this latter context it can be used to establish long time existence of transient solutions, and also to study the existence of spatially asymmetric steady solutions. Since free boundary problems are typically more challenging than their boundary-value counterparts, we shall concentrate here on a free boundary problem from developmental biology, namely, a model of tumor growth. To further exemplify the generality of our approach an instance of a boundary value problem (in a fixed domain) is presented in the last section of the paper. A variety of other problems are amenable to the same analysis, including, in particular, the Hele-Shaw model of fluid flow [11].

Within the last several decades a number of mathematical models have been developed that aimed at describing the evolution of carcinomas (see. e.g., [1, ㅎ, , 6], 8, 12, 13] and the references cited there). The main objective of these models has been to qualitatively describe, under various simplifying assumptions, the growth and stability of tumor tissue. Analysis and simulations of such models are helping to assess the relative importance of various mechanisms affecting tumor growth as well as the efficacy of certain cancer treatments. On the other hand, the description of the stationary (dormant) configurations that arise from the models has only been addressed in the case of spherical tumors, but otherwise it remains largely unexplored. In this paper we develop a method for establishing analyticity of

Received by the editors August 17, 1999.

1991 Mathematics Subject Classification. Primary 35B32, 35R35; Secondary 35B30, 35B60, 35C10, 35J85, 35Q80, 92C15, 95C15.

Key words and phrases. Free boundary problem, steady states, bifurcation, symmetrybreaking, analytic solutions, tumor growth. 
solutions of PDEs which depend on a parameter $\varepsilon$ which we use, in this context, to establish the existence of non-spherical families of dormant states for a model of non-necrotic vascularized tumors. For the sake of clarity we shall perform the analysis on two-dimensional geometries, where we demonstrate the existence of infinitely many branches of non-radially symmetric states that bifurcate from any given radial equilibrium; the solutions are analytic jointly in the spatial variables and in $\varepsilon$.

The model we shall study is a natural extension of that proposed in [7] and further analyzed in [10. We consider the tumor occupying a region $\Omega$, and denote a nutrient concentration by $\sigma$ and the internal pressure that causes the motion of cellular material by $p$. The cell proliferation rate is assumed to have the form $\mu(\sigma-\widetilde{\sigma})$ where $\mu$ and $\widetilde{\sigma}$ are positive constants. Then, after non-dimensionalization, $\sigma$ and $p$ are assumed to satisfy the diffusion equations

$$
\begin{gathered}
\Delta \sigma-\sigma=0 \text { in } \Omega, \\
\Delta p=-\mu(\sigma-\widetilde{\sigma}) \text { in } \Omega .
\end{gathered}
$$

These equations are supplemented with the boundary conditions

$$
\begin{array}{ccc}
\sigma=\bar{\sigma} & \text { on } & \partial \Omega, \\
\frac{\partial p}{\partial n}=0 & \text { on } & \partial \Omega, \\
p=\gamma \kappa & \text { on } & \partial \Omega
\end{array}
$$

where $\partial / \partial n$ denotes the normal derivative, $\kappa$ is the curvature at $\partial \Omega$, and $\bar{\sigma}, \gamma$ are positive constants.

The simplest solutions to the (free-boundary) problem (1.1)-(1.5) are the radially symmetric ones, whereby

$$
\sigma=\sigma_{0}(r), p=p_{0}(r), \Omega=\left\{r<R_{0}\right\} .
$$

As we show in $\S 3$, if $0<\widetilde{\sigma}<\frac{1}{2} \bar{\sigma}$, then there exists a unique radial solution, with $R_{0}$ depending only on $\tilde{\sigma} / \bar{\sigma}$. A natural question with obvious implications in the study of transient solutions, is whether the model admits non-radial steady states. In our search for such configurations we shall further specialize to the question of whether there exists a bifurcation branch $\left(\sigma_{\varepsilon}, \rho_{\varepsilon}, \Omega_{\varepsilon}, \gamma_{\varepsilon}\right)$ of solutions with

$$
\begin{gathered}
r=R_{0}+\varepsilon f_{1}(\theta)+\varepsilon^{2} f_{2}(\theta)+\cdots \equiv R_{0}+f(\theta, \varepsilon), \\
\sigma_{\varepsilon}(r, \theta)=\sigma_{0}(r)+\varepsilon \sigma_{1}(r, \theta)+\varepsilon^{2} \sigma_{2}(r, \theta)+\cdots, \\
p_{\varepsilon}(r, \sigma)=p_{0}(r)+\varepsilon p_{1}(r, \theta)+\varepsilon^{2} p_{2}(r, \theta)+\cdots, \\
\gamma_{\varepsilon}=\gamma_{0}+\varepsilon \gamma_{1}+\varepsilon^{2} \gamma_{2}+\cdots
\end{gathered}
$$

which bifurcates from a radial solution (1.6) for some value $\gamma=\gamma_{0}$.

We shall prove that indeed such a branch exists and that the above series are convergent and form analytic solutions in $(r, \theta, \varepsilon)$, or more precisely in $(x, y, \varepsilon)$, for $(x, y)$ in a neighborhood of $\left\{r \leq R_{0}\right\}$ and $|\varepsilon|$ small. For this we may fix $f_{1}(\theta)=$ $\cos (\ell \theta)$, for some $\ell \geq 2$, and then $\gamma_{0}$, the bifurcation point, is determined uniquely by $\mu, \bar{\sigma}, \widetilde{\sigma}$ and $\ell$; we shall actually prefer to consider $\gamma_{0}$ as a function of $R_{0}$, since we view the solutions (1.7) as a non-radial branch bifurcating from the radial solution with radius $R_{0}$. 
Let us explain the relation between this paper and general bifurcation theory. In the abstract setting of bifurcation theory one considers the problem

$$
F(\lambda, u)=0
$$

where $u$ varies in a Banach space $X$ and $\lambda$ varies in $\mathbb{R}$. Assume for simplicity that

$$
F(\lambda, 0) \equiv 0 \text { for all } \lambda \in \mathbb{R} .
$$

A bifurcation point $\left(\lambda_{c}, 0\right)$ is a point for which there exists a one- or multipleparameter branch of nontrivial solutions

$$
\lambda=\lambda(\varepsilon), u=u(\varepsilon)
$$

of (1.8) with $\lambda(0)=\lambda_{c}, u(0)=0$. The Liapounov-Schmidt procedure reduces the construction of a bifurcation branch from the infinite dimensional Banach space $X$ to a finite dimensional space:

Suppose $F(\lambda, u)$ maps $u \in X$ into a Banach space $Y$ such that $X \subset Y$, and $F$ is smooth. Set

$$
L_{0}=\frac{\partial F\left(\lambda_{c}, 0\right)}{\partial u}
$$

and assume that $L_{0}$ is a Fredholm operator with index zero. Denote its null space by $\mathcal{N}$ (we assume that $n=\operatorname{dim} \mathcal{N}<\infty$ ) and its range by $\mathcal{R}_{0}$. Let $P$ be the projection of $Y$ into $\mathcal{N}$, so that $Q=I-P$ is the projection of $Y$ into $\mathcal{R}_{0}$. If we decompose $u$ into $P u+Q u$ and project (1.8) into $\mathcal{N}$ and $\mathcal{R}_{0}$, we get the equations

$$
\begin{gathered}
K(\lambda, v, \psi) \equiv Q F(\lambda, v+\psi)=0, \\
P F(\lambda, v+\psi)=0
\end{gathered}
$$

where $v=P u$ and $\psi=Q u$. This set of equations is equivalent to the single equation (1.8). Noting that $K\left(\lambda_{c}, 0,0\right)=0$ and $\partial K\left(\lambda_{c}, 0,0\right) / \partial \psi=Q L_{0}$ is an isomorphism from $Q X$ to $Q Y$, we can apply the implicit function theorem to (1.9) and thus solve for $\psi=\psi(\lambda, v)$. We then substitute $\psi$ into (1.10) and get the Liapounov-Schmidt bifurcation equation

$$
\operatorname{PF}(\lambda, v+\psi(\lambda, v))=0
$$

which is a system of $n$ equations; for more details see, for instance, 14, 15].

The above scheme cannot be applied to the problem (1.1)-(1.5) since the spaces $X, Y$ will vary in $\varepsilon$ in a way which is unknown in advance (as it will depend on the free boundary). But even if we transform the problem to one in a fixed domain, as we shall do later on in this paper, we still cannot simply apply the above scheme, since there are no implicit function theorems that can be used to solve the resulting equation (1.9) for our problem. Thus, in a sense, our work establishes a new implicit function theorem in a space of analytic functions. The null set $\mathcal{N}$ is our case will consist of the linear space generated by $\cos (\ell \theta)$, and the orthogonality of $P u$ to $Q u$ is realized by the condition

$$
\int_{0}^{2 \pi} f_{j}(\theta) \cos \ell \theta d \theta=0 \text { for all } j \geq 2
$$

Mode 1 solutions (containing multiples of $\cos (\theta)$ in their Fourier series representations) trivially arise from infinitesimal translations of the disc $r=R_{0}$; we shall 
exclude them by imposing the conditions

$$
\begin{aligned}
& \int_{0}^{2 \pi} \sigma_{j}(r, \theta) \cos \theta d \theta=\int_{0}^{2 \pi} p_{j}(r, \theta) \cos \theta d \theta=0, \\
& \int_{0}^{2 \pi} f_{j}(\theta) \cos \theta d \theta=0 \text { for all } j \geq 1 .
\end{aligned}
$$

We shall prove that there exists, for each $\ell \geq 2$, a unique bifurcation branch of solutions (1.7) satisfying the orthogonality conditions (1.12), (1.13) and the additional condition that

$$
f_{j}(\theta), \sigma_{j}(r, \theta), p_{j}(r, \theta) \quad \text { are even functions in } \theta \text {. }
$$

The solutions are jointly analytic in the spatial variables and in $\varepsilon$. As we will show (see Remark 5.1) the orthogonality condition (1.12) implies that

$$
\gamma_{2 n+1}=0 \text { for all integers } n \geq 0 \text {; }
$$

the other coefficients $\gamma_{2 n}(n \geq 1)$ are uniquely determined by consistency conditions that need to be satisfied by the coefficients of $\cos \ell \theta$ in the $\varepsilon^{2 n+1}$ approximation.

The techniques developed in this paper depend, in part, on expanding solutions of (1.1) in a circle in terms of the modified Bessel functions $I_{n}(r)$. In $\S 2$ we introduce these Bessel functions, recall some known facts and prove new relations that will be needed in this paper. In $\S 3$ we compute the radial solution of (1.1)-(1.5). In $\S 4$ we consider the linearization of (1.1)-(1.5) about the radial solution (1.6) and prove that it has a nontrivial solution if and only if $\gamma=\gamma_{0}$ is a solution of a bifurcation equation (to be introduced later on).

In $\S 5$ we establish a formal expansion (1.7), deriving for each power $\varepsilon^{n}$ a system of equations for the coefficients $\sigma_{n}, p_{n}, f_{n}, \gamma_{n-1}$. The system has a unique solution subject to the orthogonality conditions (1.12), (1.13). The system is obtained by expressing the solution of the differential equations in terms of Bessel functions, and then expanding the boundary conditions, for the solution, in Taylor's series about $r=R_{0}$. Although this approach seems very natural, we were surprised to discover that it does not lead to a convergence proof by the standard majorization method. Indeed, in $\S 6$ we explain why a convergence proof must exploit some "hidden" cancellations in the recursive relations that determine $\sigma_{n}, p_{n}, f_{n}, \gamma_{n-1}$. We also outline in $\S 6$ another less direct and somewhat more complicated approach for deriving formal expansions. It is this approach that we shall pursue for the rest of the paper, and we briefly describe it below.

In $\S 7$ we transform the free boundary problem into a problem in the disc $\left\{r^{\prime}<1\right\}$ by a change of variables

$$
r^{\prime}=\frac{r}{R_{0}+f(\theta, \epsilon)}
$$

and set

$$
\widehat{\sigma}_{\varepsilon}(r, \theta)=\sigma_{\varepsilon}\left(r\left(R_{0}+f(\theta, \varepsilon)\right), \theta\right), \quad \hat{p}_{\varepsilon}(r, \vartheta)=p_{\varepsilon}\left(r\left(R_{0}+f(\theta, \varepsilon)\right), \theta\right) .
$$


Then $\widehat{\sigma}_{\varepsilon}, \widehat{p}_{\varepsilon}$ satisfy elliptic equations with coefficients that depend on $f, f_{\theta}, f_{\theta \theta}$. As in $\S 5$ we derive in $\S 8$ a formal expression

$$
\begin{aligned}
\widehat{\sigma}_{\varepsilon}(r, \theta) & =\widehat{\sigma}_{0}(r)+\varepsilon \widehat{\sigma}_{1}(r, \theta)+\varepsilon^{2} \widehat{\sigma}_{2}(r, \theta)+\cdots, \\
\widehat{p}_{\varepsilon}(r, \theta) & =\widehat{p}_{0}(r)+\varepsilon \widehat{p}_{1}(r, \theta)+\varepsilon^{2} \widehat{p}_{2}(r, \theta)+\cdots, \\
f(\theta, \varepsilon) & =\varepsilon f_{1}(\theta)+\varepsilon^{2} f_{2}(\theta)+\cdots, \\
\gamma & =\gamma_{0}+\varepsilon \gamma_{1}+\varepsilon^{2} \gamma_{2}+\cdots .
\end{aligned}
$$

However, from the recursive formulas for this expansion we are, in fact, able to deduce estimates that establish the convergence of the series to an analytic solution for $(x, y)$ in a neighborhood of $\{r \leq 1\}$ and $|\varepsilon|$ small; this is done in $\S \S 9-11$. In $\S 11$ we also show that $\sigma_{\varepsilon}, p_{\varepsilon}$ defined in terms of $\widehat{\sigma}_{\varepsilon}, \widehat{p}_{\varepsilon}$, by (1.16) together with $f(\theta, \varepsilon)$ and $\gamma=\gamma(\varepsilon)$ form a non-radial analytic solution of (1.1)-(1.5) jointly in the spatial variables and in $\varepsilon$ for $X=(x, y)$ in $|X| \leq R_{0}+\delta_{0},|\varepsilon| \leq \varepsilon_{0}$ and the expansion (1.7) is convergent for $0<r \leq R_{0}+\delta_{0},|\varepsilon| \leq \varepsilon_{0}$, where $\delta_{0}, \varepsilon_{0}$ are small positive constants.

Finally, in $\S 12$, we give an example of a (fixed-)boundary value problem for which our method proves joint analyticity in $(x, y, \varepsilon)$.

\section{Bessel FunCtions}

In the sequel we shall use the modified Bessel functions $I_{m}(r)$ for $m \geq 0$ and $r \geq 0$. Recall that $I_{m}(r)$ satisfies the differential equation

$$
I_{m}^{\prime \prime}(r)+\frac{1}{r} I_{m}^{\prime}(r)-\left(1+\frac{m^{2}}{r^{2}}\right) I_{m}(r)=0
$$

and is given by

$$
I_{m}(r)=\sum_{k=0}^{\infty} \frac{(r / 2)^{m+2 k}}{k ! \Gamma(m+k+1)}
$$

Furthermore,

$$
\begin{gathered}
I_{m}^{\prime}(r)+\frac{m}{r} I_{m}(r)=I_{m-1}(r), \quad m \geq 1, \\
I_{m}^{\prime}(r)-\frac{m}{r} I_{m}(r)=I_{m+1}(r),
\end{gathered}
$$

so that

$$
I_{m-1}(r)-I_{m+1}(r)=\frac{2 m}{r} I_{m}(r), \quad m \geq 1,
$$

and

$$
\begin{gathered}
r^{m+1} I_{m}(r)=\frac{d}{d r}\left(r^{m+1} I_{m+1}(r)\right), \\
I_{m}(r)=\left(\frac{1}{2 \pi r}\right)^{1 / 2} e^{r}\left[1-\frac{4 m^{2}-1}{8 r}+O\left(\frac{1}{r^{2}}\right)\right] \quad \text { if } \quad r \rightarrow \infty, \\
I_{m}(r) I_{n}(r)=\sum_{k=0}^{\infty} \frac{\Gamma(m+n+2 k+1)(r / 2)^{m+n+2 k}}{k ! \Gamma(m+k+1) \Gamma(n+k+1) \Gamma(m+n+k+1)} .
\end{gathered}
$$

In particular,

$$
I_{0}^{\prime}(r)=I_{1}(r), \quad r I_{0}(r)=\frac{d}{d r}\left(r I_{1}(r)\right)
$$


Lemma 2.1. The following inequalities hold:

$$
\begin{aligned}
& I_{m-1}(r) I_{m+1}(r)<I_{m}^{2}(r) \quad \text { if } \quad r>0, \\
& I_{m-1}(r) I_{m+1}(r)>I_{m}^{2}(r)-\frac{2}{r} I_{m}(r) I_{m+1}(r) \quad \text { if } \quad r>0 .
\end{aligned}
$$

Proof. Using (2.8) we obtain

$$
\begin{gathered}
I_{m-1}(r) I_{m+1}(r)-I_{m}^{2}(r) \\
=\sum_{k=0}^{\infty} \frac{(r / 2)^{2 m+2 k} \Gamma(2 m+2 k+1)}{k ! \Gamma(2 m+k+1)}\left[\frac{1}{\Gamma(m+k) \Gamma(m+k+2)}\right. \\
\left.-\frac{1}{(\Gamma(m+k+1))^{2}}\right], \\
\frac{2}{r} I_{m}(r) I_{m+1}(r)=\sum_{k=0}^{\infty} \frac{(r / 2)^{2 m+2 k} \Gamma(2 m+2 k+2)}{k ! \Gamma(m+k+1) \Gamma(m+k+2) \Gamma(2 m+k+2)} .
\end{gathered}
$$

To prove (2.9) it suffices to show that

$$
\frac{1}{\Gamma(m+k) \Gamma(m+k+2)}<\frac{1}{\Gamma(m+k+1)^{2}} \quad \text { if } \quad k \geq 1
$$

or

$$
\frac{1}{m+k+1}<\frac{1}{m+k}
$$

which is obvious.

To prove (2.10) it suffices to show that

$$
\begin{aligned}
& \frac{1}{\Gamma(m+k) \Gamma(m+k+2)}-\frac{1}{(\Gamma(m+k+1))^{2}} \\
& >-\frac{(2 m+2 k+1)}{\Gamma(m+k+1) \Gamma(m+k+2)(2 m+k+1)}
\end{aligned}
$$

if $k \geq 1$ (equality holds if $k=0$ ), or

$$
\begin{aligned}
& \frac{1}{\Gamma(m+k) \Gamma(m+k+1)(m+k+1)} \\
& \quad+\frac{(2 m+2 k+1)}{\Gamma(m+k+1) \Gamma(m+k)(m+k+1)(m+k)(2 m+k+1)}>\frac{1}{\Gamma(m+k+1)^{2}} .
\end{aligned}
$$

But this reduces to

$$
\frac{1}{m+k+1}+\frac{(2 m+2 k+1)}{(m+k+1)(m+k)(2 m+k+1)}>\frac{1}{m+k},
$$

or

$$
\frac{m+k}{m+k+1}+\frac{(2 m+2 k+1)}{(m+k+1)(2 m+k+1)}>1
$$

that is,

$$
\frac{2 m+2 k+1}{(m+k+1)(2 m+k+1)}>\frac{1}{m+k+1}
$$

which is obviously true. 
Corollary 2.2. If $\ell \geq 2$, then

$$
I_{2}(r)>\frac{I_{1}(r) I_{\ell+1}(r)}{I_{\ell}(r)} .
$$

Indeed, by (2.9),

$$
\frac{I_{\ell+1}(r)}{I_{\ell}(r)}<\frac{I_{\ell}(r)}{I_{\ell-1}(r)}<\cdots<\frac{I_{3}(r)}{I_{2}(r)}<\frac{I_{2}(r)}{I_{1}(r)}<\frac{I_{1}(r)}{I_{0}(r)} .
$$

Lemma 2.3. For any $r>0$, the function

$$
f(m)=\frac{I_{2}(r)-I_{1}(r) I_{m+1}(r) / I_{m}(r)}{(m-1) m(m+1)}, \quad m>1,
$$

satisfies

$$
f(m+1)<f(m) .
$$

Proof. Since

$$
f(m)=\frac{I_{2} I_{m}-I_{1} I_{m+1}}{I_{m}(m-1) m(m+1)}
$$

we need to show that

$$
\frac{I_{2} I_{m+1}-I_{1} I_{m+2}}{I_{m+1}(m+2)}<\frac{I_{2} I_{m}-I_{1} I_{m+1}}{I_{m}(m-1)}
$$

or

$$
(m-1) I_{m}\left(I_{2} I_{m+1}-I_{1} I_{m+2}\right)<(m+2) I_{m+1}\left(I_{2} I_{m}-I_{1} I_{m+1}\right) .
$$

This can be written in the form

$$
(m-1) I_{m} I_{1} I_{m+2}>(m-1) I_{m+1}^{2} I_{1}-3 I_{m+1}\left(I_{2} I_{m}-I_{1} I_{m+1}\right)
$$

or

$$
3 I_{m+1}\left(I_{2} I_{m}-I_{1} I_{m+1}\right)>I_{1}(m-1)\left(I_{m+1}^{2}-I_{m} I_{m+2}\right) .
$$

Since, by (2.10),

$$
I_{m+1}^{2}-I_{m} I_{m+2}<\frac{2}{r} I_{m+1} I_{m+2},
$$

it suffices to show that

$$
3 I_{m+1}\left(I_{2} I_{m}-I_{1} I_{m+1}\right)>I_{1}(m-1) \frac{2}{r} I_{m+1} I_{m+2} .
$$

From (2.8)

$$
\begin{aligned}
I_{2} I_{m}- & I_{1} I_{m+1} \\
= & \sum_{k=0}^{\infty} \frac{(r / 2)^{m+2+k}}{k !}\left[\frac{(m+2+2 k) !}{(m+k) !(2+k) !(m+2+k) !}\right. \\
& \left.-\frac{(m+2+2 k) !}{(m+1+k) !(1+k) !(m+2+k) !}\right] \\
= & \sum_{k=0}^{\infty} \frac{(r / 2)^{m+2+2 k}}{k !} \frac{(m+2+2 k) !}{(m+2+k) !} \frac{1}{(2+k) !(m+1+k) !}(m-1)
\end{aligned}
$$


and

$$
\frac{2}{r} I_{1} I_{m+2}=\sum_{k=0}^{\infty} \frac{(r / 2)^{m+2+2 k}}{k !} \frac{(m+3+2 k) !}{(m+2+k) !(1+k) !(m+3+k) !} .
$$

Therefore (2.13) will follow provided we have

$$
\frac{3(m+2+2 k) !(m-1)}{(m+2+k) !(2+k) !(m+1+k) !}>\frac{(m-1)(m+3+2 k) !}{(m+2+k) !(1+k) !(m+3+k) !}
$$

for all $k \geq 0$, that is, provided

$$
3(m+k+3)(m+k+2)>(m+3+2 k)(2+k) \quad \forall k \geq 0 .
$$

But since

$$
\begin{aligned}
(m+3+2 k)(2+k) & =(m+3+k)(2+k)+k(2+k) \\
& <(m+3+k)(m+k+2)+(m+3+k)(m+2+k) \\
& =2(m+k+3)(m+k+2),
\end{aligned}
$$

(2.15) is indeed valid.

Lemma 2.4. The function

$$
G_{m}(r)=r\left(\frac{I_{2}(r)}{I_{1}(r)}-\frac{I_{m+1}(r)}{I_{m}(r)}\right) \quad(m \geq 2)
$$

satisfies

$$
G_{m}^{\prime}(r)>0 \quad \text { for all } r>0 .
$$

Proof. Using (2.4) we can write

$$
G_{m}(r)=r\left(\frac{I_{1}^{\prime}}{I_{1}}-\frac{I_{m}^{\prime}}{I_{m}}\right)+m-1
$$

so that we need to show that the function

$$
F_{m}(r)=r\left(\frac{I_{1}^{\prime}}{I_{1}}-\frac{I_{m}^{\prime}}{I_{m}}\right)
$$

satisfies

$$
F_{m}^{\prime}(r)>0 \quad \text { for all } \quad r>0 .
$$

Introducing the functions

$$
U_{m}(r)=r D_{m}(r) \quad \text { where } \quad D_{m}(r)=\frac{I_{m}^{\prime}}{I_{m}},
$$

(2.19) becomes

$$
\frac{d}{d r}\left(U_{1}(r)-U_{m}(r)\right)>0 \quad \text { for all } \quad r>0
$$

By (2.1)

$$
D_{m}^{\prime}+D_{m}^{2}+\frac{1}{r} D_{m}=1+\frac{m^{2}}{r^{2}}
$$

so that

$$
r U_{m}^{\prime}+U_{m}^{2}=r^{2}+m^{2}
$$


and, by differentiation,

$$
r U_{m}^{\prime \prime}+U_{m}^{\prime}+2 U_{m} U_{m}^{\prime}=2 r .
$$

Using (2.2) we easily compute that

$$
U_{m}(r)=m+\frac{r^{2}}{2(m+1)}+O\left(r^{3}\right) \quad \text { as } \quad r \rightarrow 0 .
$$

In order to prove $(2.20)$ we first need to prove that

$$
U_{m}^{\prime}(r)>0 \quad \text { for all } \quad r>0
$$

and

$$
U_{1}(r)-U_{m}(r)<0 \quad \text { for all } r>0 .
$$

Note that these inequalities are true if $r$ is near 0 , by (2.23). Suppose first that (2.24) is not valid for all $r>0$. Then there is a smallest $r=\bar{r}$ such that

$$
U_{m}^{\prime}(\bar{r})=0
$$

and then, of course, also $U_{m}^{\prime \prime}(\bar{r}) \leq 0$. Substituting these relations into (2.22), we get a contradiction.

Similarly, if (2.25) does not hold for all $r>0$, then there is a smallest $r=\overline{\bar{r}}$ such that

$$
U_{1}(\overline{\bar{r}})-U_{m}(\overline{\bar{r}})=0
$$

then also $\left(U_{1}-U_{m}\right)^{\prime}(\overline{\bar{r}}) \geq 0$. Substituting these relations into (2.21), we again get a contradiction.

We shall now prove (2.20) by the same method. The inequality clearly holds for $r$ small, by (2.23). Hence if (2.20) is not true, then there is a smallest $r=r_{0}$ for which

$$
U_{1}^{\prime}\left(r_{0}\right)-U_{m}^{\prime}\left(r_{0}\right)=0,
$$

and then also $U_{1}^{\prime \prime}\left(r_{0}\right)-U_{m}^{\prime \prime}\left(r_{0}\right) \leq 0$. Substituting these relations into (2.22) we get

$$
U_{m} U_{m}^{\prime} \leq U_{1} U_{1}^{\prime} \quad \text { at } \quad r=r_{0}
$$

or, since $U_{1}^{\prime}\left(r_{0}\right)=U_{m}^{\prime}\left(r_{0}\right)>0$ by $(2.24)$,

$$
U_{m}\left(r_{0}\right) \leq U_{1}\left(r_{0}\right),
$$

which is a contradiction to (2.25).

Lemma 2.5. Let

$$
\begin{gathered}
g_{1 m}(r)=\frac{1}{r^{2}} \int_{0}^{r}\left[m^{2} I_{1}(s) I_{m}(s)-2 s I_{0}(s) I_{m}(s)\right] d s \\
g_{2 m}(r)=-\frac{(m-2)}{r} I_{1}(r) I_{m}(r) .
\end{gathered}
$$

Then

$$
g_{1 m}(r)+g_{2 m}(r)+\left[I_{2}(r) I_{m}(r)-I_{1}(r) I_{m+1}(r)\right]=0
$$

for any $m \geq 1$. 
Proof. Using (2.8) we get

$$
\begin{aligned}
g_{1 m}(r)= & \sum_{k=0}^{\infty} \frac{(m+2 k) !}{k !(m+k) !(k+1) !} \frac{(r / 2)^{m+2 k}}{(m+k+1) !} \frac{1}{(m+2+2 k) 2} \\
& \times\left[m^{2}(m+2 k+1)-4(k+1)(m+k+1)\right], \\
g_{2 m}(r)= & -\sum_{k=0}^{\infty} \frac{(m+1+2 k) !(r / 2)^{m+2 k}(m-2)}{k !(m+k) !(k+1) !(m+k+1) ! 2} .
\end{aligned}
$$

Hence

$$
g_{1 m}(r)+g_{2 m}(r)=\sum_{k=0}^{\infty} \frac{(r / 2)^{m+2 k}}{k !(m+k) !(k+1) !(m+k+1) !} \frac{(m+2 k) !}{(m+2+2 k) 2}[\cdots]
$$

where

$$
\begin{aligned}
{[\cdots]=} & m^{2}(m+2 k+1)-4(k+1)(m+k+1) \\
& -(m-2)(m+1+2 k)(m+2+2 k) \\
= & -2 k(m-1)(m+2 k+2) .
\end{aligned}
$$

We conclude, after changing $k$ into $k+1$, that

$$
g_{1 m}(r)+g_{2 m}(r)=-\sum_{k=0}^{\infty} \frac{(r / 2)^{m+2 k+2}}{(k+1) !(m+k+1) !} \frac{(m+2 k+2) !(k+1)(m-1)}{(k+2) !(m+k+2) !}
$$

from which (2.27) follows by using (2.14).

Corollary 2.6. For any positive integers $m, \ell, m \neq \ell$,

$$
g_{1 m}(r)+g_{2 m}(r)+\frac{m\left(m^{2}-1\right)}{\ell\left(\ell^{2}-1\right)} \frac{I_{m}(r)}{I_{\ell}(r)}\left[I_{2}(r) I_{\ell}(r)-I_{1}(r) I_{\ell+1}(r)\right] \neq 0
$$

for all $r>0$.

Proof. By Lemma 2.5 the left-hand side of (2.28) is equal to

$$
\begin{aligned}
& -\left(I_{2} I_{m}-I_{1} I_{m+1}\right)+\frac{m\left(m^{2}-1\right) I_{m}}{\ell\left(\ell^{2}-1\right) I_{\ell}}\left(I_{2} I_{\ell}-I_{1} I_{\ell+1}\right) \\
= & m\left(m^{2}-1\right) I_{m}\left\{-\frac{I_{2}-I_{1} I_{m+1} / I_{m}}{m\left(m^{2}-1\right)}+\frac{I_{2}-I_{1} I_{\ell+1} / I_{\ell}}{\ell\left(\ell^{2}-1\right)}\right\}
\end{aligned}
$$

which is $\neq 0$ by Lemma 2.3 .

\section{THE RADiAL SOLUTiON}

The radial stationary solution to $(1.2),(1.3),(1.4)$ is given by

$$
\begin{gathered}
\sigma_{0}(r)=\bar{\sigma} \frac{I_{0}(r)}{I_{0}(R)}, \quad r<R, \\
p_{0}(r)=-\mu \sigma_{0}(r)+A+\frac{\mu}{4} \tilde{\sigma} r^{2}, \quad r<R
\end{gathered}
$$

(since $\Delta\left(p_{0}+\mu \sigma_{0}\right)$ must be equal to $\left.\mu \tilde{\sigma}\right)$, where $A$ is a constant determined by (1.5):

$$
-\mu \bar{\sigma}+A+\frac{\mu \tilde{\sigma}}{4} R^{2}=\gamma \frac{1}{R}
$$


and $R$ is determined by the condition (1.4) or, equivalently, by

$$
\int_{r=R} \frac{\partial p_{0}}{\partial n}=0
$$

i.e.,

$$
0=\int_{r<R} \Delta p_{0}=-\mu \int_{r<R}\left(\sigma_{0}-\tilde{\sigma}\right) .
$$

This can be written more explicitly as

$$
\frac{2 \pi \bar{\sigma}}{I_{0}(R)} \int_{0}^{R} I_{0}(r) r d r=\tilde{\sigma} \pi R^{2}
$$

Since

$$
\begin{aligned}
\int_{0}^{R} I_{0}(r) r d r & =\int_{0}^{R} \frac{d}{d r}\left(r I_{1}(r)\right) d r \quad(\text { by }(2.6)) \\
& =R I_{1}(R)
\end{aligned}
$$

we get

$$
\frac{2 \pi \bar{\sigma}}{I_{0}(R)} R I_{1}(R)=\tilde{\sigma} \pi R^{2}
$$

Setting

$$
\Lambda=\frac{\tilde{\sigma}}{2 \bar{\sigma}}
$$

we conclude that the radius $R$ is determined by

$$
\frac{I_{1}(R)}{I_{0}(R)}=\Lambda R
$$

Theorem 3.1. If $0<\Lambda<\frac{1}{2}$, then there exists a unique solution $R=R_{0}$ to (3.4).

Proof. By (2.5)

$$
\frac{1}{R} I_{1}(R)=\frac{1}{2}\left(I_{0}(R)-I_{2}(R)\right)
$$

so that (3.4) is equivalent to

$$
\left(\frac{1}{2}-\Lambda\right) I_{0}(R)=\frac{1}{2} I_{2}(R)
$$

or

$$
\frac{I_{2}(R)}{I_{0}(R)}=1-2 \Lambda \quad(0<1-2 \Lambda<1) .
$$

Consider the function

$$
f(R)=\frac{I_{2}(R)}{I_{0}(R)}
$$

By (2.2) and (2.7),

$$
\begin{array}{ll}
f(R) \rightarrow 0 \quad \text { if } \quad R \rightarrow 0 . \\
f(R) \rightarrow 1 \quad \text { if } \quad R \rightarrow \infty .
\end{array}
$$

Therefore it suffices to show that $f(R)$ is strictly monotone increasing. But

$$
f^{\prime}=\frac{1}{I_{0}^{2}}\left(I_{2}^{\prime} I_{0}-I_{0}^{\prime} I_{2}\right)=\frac{1}{I_{0}^{2}}\left[\left(I_{1}-\frac{2}{R} I_{2}\right) I_{0}-I_{1} I_{2}\right]
$$


so that

$$
\begin{aligned}
I_{0}^{2} f^{\prime} & =\left(I_{0}-I_{2}\right) I_{1}-\frac{2}{R} I_{2} I_{0} \\
& =\frac{2}{R} I_{1}^{2}-\frac{2}{R} I_{2} I_{0}>0 \quad \text { by }(2.9) .
\end{aligned}
$$

From now on we shall always assume that

$$
0<\tilde{\sigma}<\frac{1}{2} \bar{\sigma}
$$

so that there exists a unique radial solution to (1.1)-(1.5).

Remark 3.1. Our model can be extended to transient situations by setting [10]

$$
\begin{aligned}
c \frac{\partial \sigma}{\partial t} & =\Delta \sigma-\sigma \text { in } \Omega(t), \\
\Delta p & =-\mu(\sigma-\tilde{\sigma}) \text { in } \Omega(t), \\
\frac{\partial p}{\partial n} & =-V_{n} \text { in } \partial \Omega(t)
\end{aligned}
$$

with (1.3), (1.5) on $\partial \Omega(t)$; here $V_{n}$ denotes the velocity of the free boundary $\partial \Omega(t)$. This problem was considered in 10] for 3-dimensional domains $\Omega(t)$ and solutions which are radially symmetric, i.e, $\sigma=\sigma(r, \theta), p=p(r, \theta), \Omega(t)=\{r<R(t)\}$. It was proved that if $0<\Lambda<\frac{1}{3}$, then there exists a unique stationary radial solution $\left(\sigma_{0}(r), p_{0}(r), R_{0}\right)$, and it is globally asymptotically stable with respect to the radial solutions of the time-dependent problem, provided $c$ is sufficiently small; furthermore, $\left|R(t)-R_{0}\right| \leq B e^{-\delta t}$ for all $t>0$ where $B, \delta$ are positive constants. The same result (with similar proof) is valid for the present 2-dimensional problem.

For future reference we compute

$$
\alpha=\frac{\partial \sigma_{0}}{\partial r}\left(R_{0}\right)=\bar{\sigma} \frac{I_{1}\left(R_{0}\right)}{I_{0}\left(R_{0}\right)},
$$

and

$$
\beta=-\frac{\partial^{2} p_{0}}{\partial r^{2}}\left(R_{0}\right)=\mu(\bar{\sigma}-\tilde{\sigma})=\mu \bar{\sigma}(1-2 \Lambda)
$$

or, by (3.5),

$$
\beta=\mu \bar{\sigma} \frac{I_{2}\left(R_{0}\right)}{I_{0}\left(R_{0}\right)}
$$

\section{THE LINEARIZED PROBLEM}

In this section we determine the branching points. As explained in the Introduction it will be convenient to consider the parameters $\bar{\sigma}, \tilde{\sigma}, \mu$ as fixed, and $\gamma$ as variable. We shall denote the radius $R$ of a stationary solution by $R_{0}$, and determine the parameter $\gamma=\gamma_{0}$ for which the linearized problem (about the stationary solution) has a nontrivial solution.

The radial solution is then

$$
\left(\sigma_{0}(r), p_{0}(r), R_{0}\right)
$$

and we take a perturbation of the free boundary of the form

$$
r=R_{0}+\varepsilon f_{1}(\theta)
$$


where $f_{1}(\theta)$ is of mode $\ell, \ell \geq 2$, namely,

$$
f_{1}(\theta)=N_{1} \cos \ell \theta+N_{2} \sin \ell \theta
$$

where $N_{1}, N_{2}$ are constants. By rotating the coordinate system we may assume, without loss of generality, that $N_{2}=0$. Further, by rescaling $\varepsilon$, we can choose $N_{1}=1$ so that

$$
f_{1}(\theta)=\cos \ell \theta, \quad \ell \geq 2 .
$$

Correspondingly, we take

$$
\begin{gathered}
\sigma(r, \theta)=\sigma_{0}(r)+\varepsilon \sigma_{1}(r) f_{1}(\theta), \\
p(r, \theta)-\frac{\gamma_{1}}{R_{0}}=p_{0}(r)+\varepsilon p_{1}(r) f_{1}(\theta)
\end{gathered}
$$

where the constant $\gamma_{1} / R_{0}$ arises from (1.5). We easily find that

$$
\begin{gathered}
\Delta\left(\sigma_{1} f_{1}\right)-\sigma_{1} f_{1}=0 \quad \text { if } r<R_{0} \\
\Delta\left(p_{1} f_{1}\right)=-\mu\left(\sigma_{1} f_{1}\right) \quad \text { if } r<R_{0}, \\
\sigma_{1}\left(R_{0}\right)+\alpha=0 \\
\frac{\partial p_{1}}{\partial r}\left(R_{0}\right)-\beta=0
\end{gathered}
$$

where $\alpha, \beta$ are defined in (3.7)-(3.9), and

$$
p_{1}\left(R_{0}\right)-\frac{\gamma_{0}}{R_{0}^{2}}\left(\ell^{2}-1\right)=0
$$

The last formula is obtained by using the general formula for the curvature of a curve $r=g(\theta)$,

$$
\kappa(g)=\frac{2\left(g^{\prime}\right)^{2}-g g^{\prime \prime}+g^{2}}{\left[g^{2}+\left(g^{\prime}\right)^{2}\right]^{3 / 2}}, \quad r=g(\theta)
$$

which gives, to first order in $\varepsilon$,

$$
\kappa\left(R_{0}+\varepsilon f_{1}\right)=\frac{1}{R_{0}}-\frac{\varepsilon}{R_{0}^{2}}\left(f_{1}^{\prime \prime}+f_{1}\right)=\frac{1}{R_{0}}+\frac{\varepsilon}{R_{0}^{2}}\left(\ell^{2}-1\right) f_{1} .
$$

Using this in (1.5), we easily derive the relation (4.8).

Solving for $\sigma_{1}$ from (4.4), (4.6), we get

$$
\sigma_{1}(r)=-\alpha \frac{I_{\ell}(r)}{I_{\ell}\left(R_{0}\right)} .
$$

Further, since the function

$$
M=\mu \sigma_{1}+p_{1}
$$

satisfies

$$
M^{\prime \prime}+\frac{1}{r} M^{\prime}-\frac{\ell^{2}}{r^{2}} M=0,
$$

and thus is equal to $B r^{\ell}, B$ constant, we have

$$
p_{1}(r)=B r^{\ell}+\mu \alpha \frac{I_{\ell}(r)}{I_{\ell}\left(R_{0}\right)} .
$$

The constant $B$ is determined by (4.8),

$$
B R_{0}^{\ell}=-\mu \alpha+\frac{\gamma_{0}}{R_{0}^{2}}\left(\ell^{2}-1\right)
$$


and, finally, (4.7) becomes

$$
\begin{aligned}
\beta & =\ell B R_{0}^{\ell-1}+\mu \alpha \frac{I_{\ell}^{\prime}\left(R_{0}\right)}{I_{\ell}\left(R_{0}\right)} \\
& =\frac{\ell}{R_{0}}\left[-\mu \alpha+\frac{\gamma_{0}}{R_{0}^{2}}\left(\ell^{2}-1\right)\right]+\mu \alpha \frac{I_{\ell}^{\prime}\left(R_{0}\right)}{I_{\ell}\left(R_{0}\right)} \\
& =\frac{\gamma_{0}}{R_{0}^{3}} \ell\left(\ell^{2}-1\right)+\mu \alpha\left[\frac{I_{\ell}^{\prime}\left(R_{0}\right)}{I_{\ell}\left(R_{0}\right)}-\frac{\ell}{R_{0}}\right] .
\end{aligned}
$$

Using (2.4) we get

$$
\beta=\frac{\gamma_{0}}{R_{0}^{3}} \ell\left(\ell^{2}-1\right)+\mu \alpha \frac{I_{\ell+1}\left(R_{0}\right)}{I_{\ell}\left(R_{0}\right)}
$$

which, by (3.7), (3.9), is equivalent to

$$
I_{2}\left(R_{0}\right)=\ell\left(\ell^{2}-1\right) \frac{I_{0}\left(R_{0}\right)}{\mu \bar{\sigma} R_{0}^{3}} \gamma_{0}+\frac{I_{1}\left(R_{0}\right) I_{\ell+1}\left(R_{0}\right)}{I_{\ell}\left(R_{0}\right)} .
$$

This is the bifurcation equation or the eigenvalue equation which determines $\gamma_{0}$ as a function of $R_{0}$ (and $\left.\mu, \bar{\sigma}, \tilde{\sigma}\right)$; we refer to $\gamma_{0}$ as the branching point.

Theorem 4.1. For any $R_{0}>0$ there exists a unique solution $\gamma_{0}$ of (4.13).

Proof. By Corollary 2.2

$$
I_{2}\left(R_{0}\right)=\frac{I_{1}\left(R_{0}\right) I_{\ell+1}\left(R_{0}\right)}{I_{\ell}\left(R_{0}\right)}+q
$$

where $q$ is a positive number. Hence the unique solution of (4.13) is given by

$$
\ell\left(\ell^{2}-1\right) \frac{I_{0}\left(R_{0}\right)}{\mu \bar{\sigma} R_{0}^{3}} \gamma_{0}=q .
$$

Remark 4.1. For $\ell=1$, (4.13) holds identically for all $\gamma_{0}, R_{0}$. The reason is that any $\varepsilon$-translation of the radial solution represents an $\varepsilon$-perturbation of mode $\ell=1$ when viewed from the original system of coordinates. More precisely, the radial solution (with radius $R_{0}$ ) with respect to the center $x_{1}=\varepsilon, x_{2}=0$, when written in polar coordinates centered at $x_{1}=0, x_{2}=0$, has the form

$$
r=f(\theta, \varepsilon)=R_{0}+\varepsilon R_{0} \cos \theta+O\left(\varepsilon^{2}\right) .
$$

Later on we shall construct bifurcation branches with free boundary

$$
r=R_{0}+\varepsilon \cos \ell \theta+\sum_{n \geq 2} \varepsilon^{n} \lambda_{n}(\theta)
$$

uniquely determined by the orthogonality conditions $\int_{0}^{2 \pi} \lambda_{n}(\theta) \cos \ell \theta d \theta=0$. For $\ell \geq 2$ such a curve is not radially symmetric if $|\varepsilon|$ is small enough. However, for $\ell=1$ this curve may correspond to the curve obtained above by $\varepsilon$-translation of a radial solution. Since we are interested in symmetry-breaking bifurcations, we restrict ourselves to $\ell \geq 2$.

From (3.3), (3.4) we have

$$
\bar{\sigma}=\frac{\tilde{\sigma}}{2 \Lambda}=\frac{\tilde{\sigma}}{2} R_{0} \frac{I_{0}\left(R_{0}\right)}{I_{1}\left(R_{0}\right)}
$$


so that the eigenvalue equation (4.13) can be written in the form

$$
H\left(R_{0}\right) \equiv R_{0}^{4}\left[\frac{I_{2}\left(R_{0}\right)}{I_{1}\left(R_{0}\right)}-\frac{I_{\ell+1}\left(R_{0}\right)}{I_{\ell}\left(R_{0}\right)}\right]=N
$$

where

$$
N=\frac{1}{\mu \tilde{\sigma}} 2 \ell\left(\ell^{2}-1\right) \gamma_{0}
$$

By Lemma $2.4, H\left(R_{0}\right)$ is strictly increasing in $R_{0}$, and in fact $H^{\prime}\left(R_{0}\right)>0$, so that for each $\gamma_{0}$ there is a unique $R_{0}$ satisfying (4.13). From (2.2) we easily deduce that $H\left(R_{0}\right) \rightarrow 0$ if $R_{0} \rightarrow 0$, and from (2.7) we deduce that $H\left(R_{0}\right) \rightarrow \infty$ if $R_{0} \rightarrow \infty$. Hence we have

Theorem 4.2. The function $\gamma_{0}=\gamma\left(R_{0}\right)$ determined by the eigenvalue equation (4.13) satisfies

$$
\begin{gathered}
\gamma^{\prime}\left(R_{0}\right)>0, \\
\lim _{R_{0} \rightarrow 0} \gamma\left(R_{0}\right)=0, \quad \lim _{R_{0} \rightarrow \infty} \gamma\left(R_{0}\right)=\infty .
\end{gathered}
$$

In $\S 8$ we shall encounter linear systems of two equations with coefficient matrices $A_{m}$, where

$$
\operatorname{det} A_{m}=\mu \tilde{\sigma} R_{0}+\frac{\mu}{I_{m}\left(R_{0}\right)} \int_{0}^{1} I_{m}\left(R_{0} \tau\right) g_{m}(\tau) d \tau-m\left(\mu \frac{\tilde{\sigma}}{2} R_{0}-\frac{\gamma_{0}}{R_{0}^{2}}\left(m^{2}-1\right)\right)
$$

with

$$
g_{m}(\tau)=\left[m^{2} I_{1}\left(R_{0} \tau\right)-2 R_{0} \tau I_{0}\left(R_{0} \tau\right)\right] \frac{\bar{\sigma}}{I_{0}\left(R_{0}\right)} .
$$

Using the relation $\tilde{\sigma} / \bar{\sigma}=2 I_{1}\left(R_{0}\right) /\left(R_{0} I_{0}\left(R_{0}\right)\right)$ (see $\left.(3.3),(3.4)\right)$ we can write

$$
\begin{aligned}
\operatorname{det} A_{m}= & \frac{\mu \bar{\sigma} R_{0}}{I_{0}\left(R_{0}\right) I_{m}\left(R_{0}\right)}\left\{-\frac{(m-2)}{R_{0}} I_{1}\left(R_{0}\right) I_{m}\left(R_{0}\right)\right. \\
& \left.+\frac{1}{R_{0}^{2}} \int_{0}^{R_{0}}\left[m^{2} I_{1}(\tau)-2 \tau I_{0}(\tau)\right] I_{m}(\tau) d \tau+\frac{\gamma_{0} m\left(m^{2}-1\right)}{\mu \bar{\sigma} R_{0}^{3}} I_{0}\left(R_{0}\right) I_{m}\left(R_{0}\right)\right\}
\end{aligned}
$$

Substituting $\gamma_{0}$ from (4.13) and using Lemma 2.5 and Corollary 2.6, we get

Lemma 4.3. If $\operatorname{det} A_{m}$ is defined by (4.17), (4.18), then

$$
\operatorname{det} A_{\ell}=0, \quad \text { and } \quad \operatorname{det} A_{m} \neq 0 \text { for all } m \neq \ell .
$$




\section{Formal EXPANSION}

In this section we derive the formal expansion (1.7) of the bifurcation branch near $\gamma=\gamma_{0}$ in the following form:

$$
\begin{gathered}
\sigma=\sigma(r, \theta, \varepsilon)=\sum_{n \geq 0} \sigma_{n}(r, \theta) \varepsilon^{n}=\sigma_{0}(r)+\sum_{n \geq 1}\left(\sum_{k \geq 0} \sigma_{n k}(\theta)\left(r-R_{0}\right)^{k}\right) \varepsilon^{n} \\
p=p(r, \theta, \varepsilon)=\sum_{n \geq 0} p_{n}(r, \theta) \varepsilon^{n}=p_{0}(r)+\sum_{n \geq 1}\left(\sum_{k \geq 0} p_{n k}(\theta)\left(r-R_{0}\right)^{k}\right) \varepsilon^{n} \\
r=R_{0}+f(\theta, \varepsilon)=R_{0}+\varepsilon \cos \ell \theta+\sum_{n \geq 2} f_{n}(\theta) \varepsilon^{n}, \\
\gamma=\gamma(\varepsilon)=\gamma_{0}+\sum_{n \geq 1} \gamma_{n} \varepsilon^{n}
\end{gathered}
$$

where the radius $R_{0}$ and the integer $\ell$ are arbitrarily fixed. The functions $\sigma$ and $p$ must satisfy the equations (1.2) and (1.3) subject to the boundary conditions

$$
\sigma\left(R_{0}+f(\theta, \varepsilon), \theta, \varepsilon\right)=\bar{\sigma}, \quad \frac{\partial p}{\partial n_{\varepsilon}}\left(R_{0}+f(\theta, \varepsilon), \theta, \varepsilon\right)=0
$$

and

$$
p\left(R_{0}+f(\theta, \varepsilon), \theta, \varepsilon\right)=\gamma(\varepsilon) \kappa_{\varepsilon}
$$

where $n_{\varepsilon}$ and $\kappa_{\varepsilon}$ denote the unit normal vector and curvature of the boundary $\left\{r=R_{0}+f(\theta, \varepsilon)\right\}$, respectively.

Recall that the curvature $\kappa(g)$ of a curve $r=g(\theta)$ is given by (4.9) and note that

$$
\frac{\partial}{\partial n}=\frac{1}{\left(g^{2}+\left(g^{\prime}\right)^{2}\right)^{1 / 2}}\left(g \frac{\partial}{\partial r}-\frac{g^{\prime}}{g} \frac{\partial}{\partial \theta}\right)
$$

along the curve $r=g(\theta)$. Hence the boundary conditions (1.3)- (1.5) can be written in the form

$$
\begin{gathered}
\sum_{n \geq 1} \varepsilon^{n} \sum_{k \geq 0} \sigma_{n k}(\theta)\left(\sum_{i \geq 1} f_{i}(\theta) \varepsilon^{i}\right)^{k}+\sum_{k \geq 1} \sigma_{0 k}\left(\sum_{i \geq 1} f_{i}(\theta) \varepsilon^{i}\right)^{k}=0 \\
\left(R_{0}+f(\theta, \varepsilon)\right)^{2} \frac{\partial p}{\partial r}\left(R_{0}+f(\theta, \varepsilon), \theta, \varepsilon\right)-f^{\prime}(\theta, \varepsilon) \frac{\partial p}{\partial \theta}\left(R_{0}+f(\theta, \varepsilon), \theta, \varepsilon\right)=0
\end{gathered}
$$

or

$$
\begin{gathered}
\left(R_{0}+\sum_{i \geq 1} f_{i} \varepsilon^{i}\right)^{2}\left\{\sum_{n \geq 1} \varepsilon^{n} \sum_{k \geq 1} k p_{n k}(\theta)\left(\sum_{i \geq 1} f_{i} \varepsilon^{i}\right)^{k-1}+\sum_{k \geq 2} k p_{0 k}\left(\sum_{i \geq 1} f_{i} \varepsilon^{i}\right)^{k-1}\right\} \\
-\left(\sum_{i \geq 1} f_{i}^{\prime} \varepsilon^{i}\right) \sum_{n \geq 1} \varepsilon^{n}\left[\sum_{k \geq 0} p_{n k}^{\prime}(\theta)\left(\sum_{i \geq 1} f_{i} \varepsilon^{i}\right)^{k}\right]=0
\end{gathered}
$$

and

$$
\begin{aligned}
& {\left[\left(R_{0}+f(\theta, \varepsilon)\right)^{2}+\left(f^{\prime}(\theta, \varepsilon)\right)^{2}\right]^{3 / 2} p\left(R_{0}+f(\theta, \varepsilon), \theta, \varepsilon\right)} \\
& \quad=\gamma(\varepsilon)\left[\left(R_{0}+f(\theta, \varepsilon)\right)^{2}+2\left(f^{\prime}(\theta, \varepsilon)\right)^{2}-\left(R_{0}+f(\theta, \varepsilon)\right) f^{\prime \prime}(\theta, \varepsilon)\right]
\end{aligned}
$$


or

$$
\left[\left(R_{0}+\sum_{i \geq 1} f_{i} \varepsilon^{i}\right)^{2}+\left(\sum_{i \geq 1} f_{i}^{\prime} \varepsilon^{i}\right)^{2}\right]^{3 / 2} \sum_{n \geq 0} \varepsilon^{n}\left[\sum_{k \geq 0} p_{n k}\left(\sum_{i \geq 1} f_{i} \varepsilon^{i}\right)^{k}\right]
$$

$$
=\left(\sum_{i \geq 0} \gamma_{i} \varepsilon^{i}\right)\left[\left(R_{0}+\sum_{i \geq 1} f_{i} \varepsilon^{i}\right)^{2}+2\left(\sum_{i \geq 1} f_{i}^{\prime} \varepsilon^{i}\right)^{2}-\left(R_{0}+\sum_{i \geq 1} f_{i} \varepsilon^{i}\right)\left(\sum_{i \geq 1} f_{i}^{\prime \prime} \varepsilon^{i}\right)\right] .
$$

Comparing the coefficients of $\varepsilon^{n}$ we get

$$
\sigma_{n 0}+\sigma_{01} f_{n}(\theta)=F^{1}(\theta) \quad\left(\sigma_{01}=\alpha ; \text { cf. }(3.7)\right)
$$

where

$$
\begin{gathered}
F^{1}(\theta)=-\left.\frac{1}{n !} \partial_{\varepsilon}^{n}\right|_{\varepsilon=0}\left[\sum_{m=1}^{n-1} \varepsilon^{m} \sum_{k \geq 0} \sigma_{m k}(\theta)\left(\sum_{i \geq 1} f_{i} \varepsilon^{i}\right)^{k}+\sum_{k \geq 2} \sigma_{0 k}\left(\sum_{i \geq 1} f_{i} \varepsilon^{i}\right)^{k}\right], \\
p_{n 1}+2 p_{02} f_{n}=F^{2}(\theta) \quad\left(2 p_{02}=-\beta ; \text { cf. }(3.9)\right)
\end{gathered}
$$

where

$$
\begin{aligned}
F^{2}(\theta) & =-\left.\frac{1}{R_{0}^{2}} \frac{1}{n !} \partial_{\varepsilon}^{n}\right|_{\varepsilon=0}\left[( R _ { 0 } + \sum _ { i \geq 1 } f _ { i } \varepsilon ^ { i } ) ^ { 2 } \sum _ { m = 1 } ^ { n - 1 } \varepsilon ^ { m } \left[\sum_{k \geq 1} k p_{m k}\left(\sum_{i \geq 1} f_{i} \varepsilon^{i}\right)^{k-1}\right.\right. \\
& \left.\left.+\sum_{k \geq 3} k p_{0 k}\left(\sum_{i \geq 1} f_{i} \varepsilon^{i}\right)\right]-\left(\sum_{i \geq 1} f_{i}^{\prime} \varepsilon^{i}\right) \sum_{m=1}^{n-1} \varepsilon^{m} \sum_{k \geq 0} p_{m k}^{\prime}\left(\sum_{i \geq 1} f_{i} \varepsilon^{i}\right)^{k}\right]
\end{aligned}
$$

and, writing $p_{n-1,0}=\left(p_{n-1,0}-\frac{\gamma_{n-1}}{R_{0}}\right)+\frac{\gamma_{n-1}}{R_{0}}$ in $(5.7)$,

$$
\begin{aligned}
& \frac{\gamma_{n-1}}{R_{0}} \frac{3}{R_{0}} f_{1}+p_{n 0}+\frac{3}{R_{0}} f_{n} p_{00}=\frac{\gamma_{n}}{R_{0}}+\frac{\gamma_{0}}{R_{0}^{3}}\left(2 R_{0} f_{n}-R_{0} f_{n}^{\prime \prime}\right) \\
& \quad+\frac{\gamma_{n-1}}{R_{0}^{3}}\left(2 R_{0} f_{1}-R_{0} f_{1}^{\prime \prime}\right)+F^{3}(\theta) \quad\left(p_{00}=\frac{\gamma_{0}}{R_{0}}, p_{01}=0\right)
\end{aligned}
$$

where

$$
\begin{aligned}
F_{3}(\theta) & =\left.\frac{1}{R_{0}^{3}} \frac{1}{n !} \partial_{\varepsilon}^{n}\right|_{\varepsilon=0}\left\{( \sum _ { i = 1 } ^ { n - 2 } \gamma _ { i } \varepsilon ^ { i } ) \left[\left(R_{0}+\sum_{i=1}^{n-1} f_{i} \varepsilon^{i}\right)^{2}+2\left(\sum_{i=1}^{n-1} f_{i}^{\prime} \varepsilon^{i}\right)^{2}\right.\right. \\
- & \left.\left(R_{0}+\sum_{i=1}^{n-1} f_{i} \varepsilon^{i}\right)\left(\sum_{i=1}^{n-1} f_{i}^{\prime \prime} \varepsilon^{i}\right)\right]-\left[\left(R_{0}+\sum_{i=1}^{n-1} f_{i} \varepsilon^{i}\right)^{2}+\left(\sum_{i=1}^{n-1} f_{i}^{\prime} \varepsilon^{i}\right)^{2}\right]^{3 / 2} \\
\times & {\left.\left[\sum_{m=0}^{n-2} \varepsilon^{m} \sum_{k \geq 0} p_{m k}\left(\sum_{i \geq 1} f_{i} \varepsilon^{i}\right)^{k}+\varepsilon^{n-1}\left(\left(p_{n-1,0}-\frac{\gamma_{n-1}}{R_{0}}\right)+p_{n-1,1} f_{1} \varepsilon\right)\right]\right\} }
\end{aligned}
$$

Thus, $\left(\sigma_{n}, p_{n}, f_{n}\right)$ will satisfy

$$
\Delta \sigma_{n}-\sigma_{n}=0, \quad \Delta p_{n}=-\mu \sigma_{n} \quad \text { in } \quad r<R_{0}
$$


and, from (5.8)-(5.10),

$$
\begin{gathered}
\sigma_{n}\left(R_{0}, \theta\right)+\alpha f_{n}(\theta)=F^{1}(\theta), \\
\frac{\partial p_{n}}{\partial r}\left(R_{0}, \theta\right)-\beta f_{n}(\theta)=F^{2}(\theta), \\
p_{n}\left(R_{0}, \theta\right)+\frac{\gamma_{0}}{R_{0}^{2}}\left(f_{n}+f_{n}^{\prime \prime}\right)=\frac{\gamma_{n}}{R_{0}}+\frac{\gamma_{n-1}}{R_{0}^{2}}\left(\ell^{2}-1\right) \cos \ell \theta+F^{3}(\theta) .
\end{gathered}
$$

We shall inductively show that functions $\sigma_{n}(r, \theta), p_{n}(r, \theta)-\gamma_{n} / R_{0}$ and $f_{n}(\theta)$ together with numbers $\gamma_{n-1}$ can be chosen so as to satisfy (5.11)-(5.12) for each $n$ and this choice is unique under the orthogonality condition (1.12), (1.13) and the condition (1.14).

Thus we assume that for $m<n, f_{m}, p_{m 0}-\gamma_{m} / R_{0}, \sigma_{m 0}$, and $p_{m k}, \sigma_{m k}(k \geq 1)$ have been determined and prove that the functions $f_{n}, p_{n 0}-\gamma_{n} / R_{0}, \sigma_{n 0}, p_{n h}(h \geq 1)$, $\sigma_{n h}(h \geq 1)$ and the constant $\gamma_{n-1}$ are uniquely determined (subject to the orthogonality conditions (1.12), (1.13) and the condition (1.14)). Note that once $\gamma_{n-1}$ is determined, so is also $p_{n-1,0}$ as the difference $p_{n-1,0}-\gamma_{n-1} / R_{0}$ was already determined inductively.

The inductive proof follows from the following general lemma.

Lemma 5.1. Let $F^{1} \in C^{2+\alpha}, F^{2} \in C^{1+\alpha}, F^{3} \in C^{\alpha}$ be even functions of $\theta$ satisfying

$$
\int_{0}^{2 \pi} F^{i}(\theta) \cos \theta d \theta=0
$$

and consider the problem

$$
\Delta \sigma-\sigma=0, \quad \Delta p=-\mu \sigma \quad \text { in } \quad r<R_{0}
$$

with boundary conditions

$$
\begin{gathered}
\sigma+\alpha f=F^{1}, \quad \frac{\partial p}{\partial r}-\beta f=F^{2}, \\
p+\frac{\gamma_{0}}{R_{0}^{2}}\left(f+f^{\prime \prime}\right)=F^{3}+\frac{\gamma}{R_{0}^{2}}\left(\ell^{2}-1\right) \cos \ell \theta .
\end{gathered}
$$

Then there exists a unique solution $(\sigma, p, f, \gamma)$ whose components are even functions of $\theta$ and such that

$$
\begin{aligned}
& \int_{0}^{2 \pi} f(\theta) \cos m \theta d \theta=0 \quad \text { for } \quad m=1, \ell, \\
& \int_{0}^{2 \pi} \sigma(r, \theta) \cos \theta d \theta=\int_{0}^{2 \pi} p(r, \theta) \cos \theta d \theta=0 ;
\end{aligned}
$$

moreover,

$$
\begin{gathered}
|\sigma|_{2+\alpha}+|p|_{2+\alpha}+|f|_{2+\alpha} \leq C\left(\left|F^{1}\right|_{2+\alpha}+\left|F^{2}\right|_{1+\alpha}+\left|F^{3}\right|_{\alpha}\right) \text { and } \\
|\gamma| \leq C \sum_{i=1}^{3}\left|\int_{0}^{2 \pi} F^{i}(\theta) \cos \ell \theta d \theta\right| .
\end{gathered}
$$

Here $|\sigma|_{2+\alpha}$ and $|p|_{2+\alpha}$ are the $C^{2+\alpha}$ norms in the disc $r<R_{0}$, whereas $|f|_{2+\alpha}$ is the $C^{2+\alpha}$ norm with respect to $\theta$. 
Proof. We first establish uniqueness. Since the $F^{i}$ and $\sigma, p, f$ are even functions of $\theta$, it follows from (5.13) and (5.14) that

$$
\begin{aligned}
\sigma & =\sum_{m \neq 1} A_{m} \cos m \theta \cdot I_{m}(r), \\
p & =\sum_{m \neq 1}\left[B_{m} \cos m \theta \cdot r^{m}-\mu A_{m} \cos m \theta \cdot I_{m}(r)\right], \\
f & =\sum_{m \neq 1} \tau_{m} \cos m \theta
\end{aligned}
$$

and

$$
F^{i}=\sum_{m \neq 1} F_{m}^{i} \cos m \theta
$$

we do not impose as yet the orthogonality condition $\int_{0}^{2 \pi} f(\theta) \cos \ell \theta d \theta=0$.

The boundary conditions then yield, for $m \neq \ell$,

$$
\begin{array}{r}
A_{m} I_{m}\left(R_{0}\right)+\alpha \tau_{m}=F_{m}^{1}, \\
-\mu A_{m} I_{m}^{\prime}\left(R_{0}\right)+B_{m} R_{0}^{m-1} m-\beta \tau_{m}=F_{m}^{2}, \\
-\mu A_{m} I_{m}\left(R_{0}\right)+B_{m} R_{0}^{m}-\frac{\gamma_{0}}{R_{0}^{2}}\left(m^{2}-1\right) \tau_{m}=F_{m}^{3},
\end{array}
$$

or

$$
T_{m}\left(\begin{array}{c}
A_{m} \\
B_{m} \\
\tau_{m}
\end{array}\right)=\left(\begin{array}{c}
F_{m}^{1} \\
F_{m}^{2} \\
F_{m}^{3}
\end{array}\right)
$$

where $T_{m}$ is given by

$$
T_{m}=\left(\begin{array}{ccc}
I_{m}\left(R_{0}\right) & 0 & \alpha \\
-\mu I_{m}^{\prime}\left(R_{0}\right) & m R_{0}^{m-1} & -\beta \\
-\mu I_{m}\left(R_{0}\right) & R_{0}^{m} & -\frac{\gamma_{0}}{R_{0}^{2}}\left(m^{2}-1\right)
\end{array}\right) .
$$

Lemma 5.2. There holds

$$
\operatorname{det} T_{1}=0, \quad \operatorname{det} T_{\ell}=0
$$

and

$$
\operatorname{det} T_{m} \neq 0 \quad \text { if } \quad m \neq 1, m \neq \ell
$$

furthermore,

$$
\left|\operatorname{det} T_{m}\right| \geq C_{1} R_{0}^{m} m^{3} I_{m}\left(R_{0}\right) \quad \forall m \neq 1, \ell
$$

where $C_{1}$ is a positive constant independent of $m$.

Proof. Adding $\left(-m / R_{0}\right) \times($ third row) to the second row we get (cf. (2.4))

$$
\begin{aligned}
-R_{0}^{-m} \operatorname{det} T_{m} & =\left|\begin{array}{cc}
I_{m}\left(R_{0}\right) & \alpha \\
-\mu I_{m+1}\left(R_{0}\right) & -\beta+\frac{\gamma_{0}}{R_{0}^{3}} m\left(m^{2}-1\right)
\end{array}\right| \\
& =I_{m}\left(R_{0}\right)\left\{-\beta+\frac{\gamma_{0}}{R_{0}^{3}} m\left(m^{2}-1\right)+\mu \alpha \frac{I_{m+1}\left(R_{0}\right)}{I_{m}\left(R_{0}\right)}\right\} .
\end{aligned}
$$


By (3.7), (3.9) the function in braces is equal to

$$
\frac{\mu \bar{\sigma}}{I_{0}\left(R_{0}\right)}\left\{m\left(m^{2}-1\right) \frac{I_{0}\left(R_{0}\right)}{\mu \bar{\sigma} R_{0}^{3}} \gamma_{0}+\frac{I_{1}\left(R_{0}\right) I_{m+1}\left(R_{0}\right)}{I_{m}\left(R_{0}\right)}-I_{2}\left(R_{0}\right)\right\}
$$

and by (4.13) it is equal to zero if $m=\ell$; it is clearly also equal to zero if $m=1$. Note also that the expression in braces is $\neq 0$ if $m=0$ since $I_{1}^{2} / I_{0} \neq I_{2}$ (by Lemma 2.1 ), and so $\operatorname{det} T_{0} \neq 0$.

Recalling the definition of $f(m)$ in $(2.12)$ we can write, for $m \neq 0,1$,

$$
\left|\operatorname{det} T_{m}\right|=R_{0}^{m} I_{m}\left(R_{0}\right) \frac{\mu \bar{\sigma}}{I_{0}\left(R_{0}\right)} m\left(m^{2}-1\right)\left|f(m)-\frac{\gamma_{0} I_{0}\left(R_{0}\right)}{\mu \bar{\sigma} R_{0}^{3}}\right| \text {. }
$$

Using Lemma 2.3 we conclude that (5.19) holds. Furthermore, since $I_{m+1}(r)<$ $\frac{r}{2} I_{m}(r), f(m) \rightarrow 0$ if $m \rightarrow \infty$ and, consequently, (5.20) is valid.

Returning to the proof of Lemma 5.1 we note that from the last two equations in (5.16) it follows that

$$
\left[-\mu I_{m}^{\prime}\left(R_{0}\right) R_{0}+\mu I_{m}\left(R_{0}\right) m\right] A_{m}+\left[m\left(m^{2}-1\right) \frac{\gamma}{R_{0}^{2}}-\beta R_{0}\right] \tau_{m}=F_{m}^{2} R_{0}-m F_{m}^{3}
$$

and, by (2.4), the coefficient of $A_{m}$ is equal to $-\mu R_{0} I_{m+1}\left(R_{0}\right)$. Solving this equation together with the first equation in (5.16) we find that, for $m \geq 1$,

$$
\begin{aligned}
\left|A_{m}\right| & \leq \frac{R_{0}^{m-1}}{\left|\operatorname{det} T_{m}\right|}\left|\left[m\left(m^{2}-1\right) \frac{\gamma}{R_{0}^{2}}-\beta R_{0}\right] F_{m}^{1}-\alpha\left(F_{m}^{2} R_{0}-m F_{m}^{3}\right)\right| \\
& \leq \frac{C}{\left|I_{m}\right|}\left[\left|F_{m}^{1}\right|+\frac{\left|F_{m}^{2}\right|}{m^{3}}+\frac{\left|F_{m}^{3}\right|}{m^{2}}\right], \\
\left|\tau_{m}\right| & \leq C\left[\left|F_{m}^{1}\right| \frac{\left|I_{m+1}\right|}{\left|I_{m}\right| m^{3}}+\frac{\left|F_{m}^{2}\right|}{m^{3}}+\frac{\left|F_{m}^{3}\right|}{m^{2}}\right] .
\end{aligned}
$$

Finally, the third equation in (5.16) gives

$$
\begin{aligned}
\left|B_{m}\right| & \leq \frac{C}{R_{0}^{m}}\left[\left|A_{m}\right| I_{m}+m^{2}\left|\tau_{m}\right|+\left|F_{m}^{3}\right|\right] \\
& \leq \frac{C}{R_{0}^{m}}\left[\left|F_{m}^{1}\right|+\left|F_{m}^{1}\right| \frac{I_{m+1}}{I_{m} m}+\frac{\left|F_{m}^{2}\right|}{m}+\left|F_{m}^{3}\right|\right] .
\end{aligned}
$$

Using the relation [16, p. 225]

$$
I_{m}(z)=i^{-m} \sqrt{\frac{1}{2 \pi m}}\left(\frac{e i z}{2 m}\right)^{m}\left(1+O\left(\frac{1}{m}\right)\right) \quad m \rightarrow \infty,
$$

if we write

$$
\begin{aligned}
\sigma & =\sum_{m \neq 1} \tilde{A}_{m} \cos m \theta \frac{I_{m}(r)}{I_{m}\left(R_{0}\right)} \\
p & =\sum_{m \neq 1}\left[\tilde{B}_{m} \cos m \theta\left(\frac{r}{R_{0}}\right)^{m}-\mu \tilde{A}_{m} \cos m \theta \frac{I_{m}(r)}{I_{m}\left(R_{0}\right)}\right], \\
f & =\sum_{m \neq 1} \tilde{\tau}_{m} \cos m \theta
\end{aligned}
$$


then, for $m \neq \ell$,

$$
\begin{gathered}
\left|\tilde{A}_{m}\right| \leq C\left[\left|F_{m}^{1}\right|+\frac{\left|F_{m}^{2}\right|}{m^{3}}+\frac{\left|F_{m}^{3}\right|}{m^{2}}\right], \\
\left|\tilde{B}_{m}\right| \leq C\left[\left|F_{m}^{1}\right|+\frac{F_{m}^{2}}{m}+\left|F_{m}^{3}\right|\right], \\
\left|\tilde{\tau}_{m}\right| \leq C\left[\frac{\left|F_{m}^{1}\right|}{m^{4}}+\frac{\left|F_{m}^{2}\right|}{m^{3}}+\frac{\left|F_{m}^{3}\right|}{m^{2}}\right] .
\end{gathered}
$$

In case $m=\ell$ we have det $T_{\ell}=0$ and the solvability condition becomes

$$
F_{\ell}^{1}\left[\ell\left(\ell^{2}-1\right) \frac{\gamma_{0}}{R_{0}^{2}}-\beta R_{0}\right]=\alpha\left[F_{\ell}^{2} R_{0}-\ell F_{\ell}^{3}-\ell\left(\ell^{2}-1\right) R_{0}^{-2} \gamma\right] .
$$

This defines $\gamma$ uniquely, and also establishes the last estimate in (5.15). However, the system for $\left(A_{\ell}, B_{\ell}, \tau_{\ell}\right)$ has infinitely many solutions, obtained by taking a special solution and adding a multiple of $\cos \ell \theta$. By imposing the orthogonality condition

$$
\int_{0}^{2 \pi} f(\theta) \cos \ell \theta d \theta=0
$$

we get uniqueness. The orthogonality condition means that $\widetilde{\tau}_{\ell}=0$, and then

or

$$
A_{\ell}=\frac{F_{\ell}^{1}}{I_{\ell}\left(R_{0}\right)}, \quad B_{\ell}=\left(F_{\ell}^{3}+\mu F_{\ell}^{1}\right) / R_{0}^{\ell},
$$

$$
\tilde{A}_{\ell}=F_{\ell}^{1}, \quad \tilde{B}_{\ell}=F_{\ell}^{3}+\mu F_{\ell}^{1}, \quad \tilde{\tau}_{\ell}=0 .
$$

The above proof actually establishes also existence in the $L^{2}$ framework. Indeed, with $\tilde{A}_{m}, \tilde{B}_{m}, \tilde{\tau}_{m}$ defined as above, the estimates (5.23) show that $(\sigma, p, f)$ form a solution in a weak sense, and

$$
\int_{0}^{2 \pi}\left[\left|\sigma\left(R_{0}, \theta\right)\right|^{2}+\left|p\left(R_{0}, \theta\right)\right|^{2}\right] d \theta \leq C \int_{0}^{2 \pi} \sum\left(F^{i}\right)^{2} d \theta
$$

We shall now establish the $(2+\alpha)$-estimates asserted in (5.15).

By Schauder's estimate

$$
|\sigma|_{2+\alpha}+|p|_{2+\alpha} \leq C\left(\left|F^{1}\right|_{2+\alpha}+\left|F^{2}\right|_{1+\alpha}+|f|_{2+\alpha}+|p|_{0}\right) .
$$

On the other hand, from the third boundary condition for the system satisfied by $(\sigma, p, f, \gamma)$ we deduce that

$$
f(\theta)=\int_{0}^{\theta} \sin (\theta-s) h(s) d s+a \cos \theta+b \sin \theta
$$

where

$$
h(s)=\left[F^{3}(s)+\frac{\gamma}{R_{0}^{2}}\left(\ell^{2}-1\right) \cos \ell s-p\left(R_{0}, s\right)\right] \frac{R_{0}^{2}}{\gamma_{0}} .
$$

Since

$$
\int_{0}^{2 \pi} h(s)\left(\begin{array}{c}
\sin s \\
\cos s
\end{array}\right) d s=0,
$$

the requirement that

$$
\int_{0}^{2 \pi} f(\theta)\left(\begin{array}{c}
\sin \theta \\
\cos \theta
\end{array}\right) d \theta=0
$$


gives

$$
\left(\begin{array}{l}
a \\
b
\end{array}\right)=-\frac{1}{2 \pi} \int_{0}^{2 \pi} s h(s)\left(\begin{array}{c}
\sin s \\
\cos s
\end{array}\right) d s .
$$

Using this in (5.27) we can estimate $f$ :

$$
|f|_{2+\alpha} \leq C|h|_{\alpha} \leq C\left[\left|F^{3}\right|_{\alpha}+|\gamma|+\left|p\left(R_{0}, \cdot\right)\right|_{\alpha}\right] .
$$

Recalling the estimate (5.15) on $\gamma$ and using also the inequality

$$
\left|p\left(R_{0}, \cdot\right)\right|_{\alpha} \leq \varepsilon|p|_{2+\alpha}+C\left|p\left(R_{0}, \cdot\right)\right|_{L^{2}} \quad \forall \varepsilon>0,
$$

and (5.25), we conclude that

$$
|f|_{2+\alpha} \leq C\left[\left|F^{3}\right|_{\alpha}+\left|F^{1}\right|_{0}+\left|F^{2}\right|_{0}+\varepsilon|p|_{2+\alpha}\right] .
$$

If we substitute this into (5.26) and again use (5.25), we obtain the assertion (5.15).

Remark 5.1. From the second inequality in (5.15) it follows that $\gamma_{2 k+1}=0$ for all integers $k \geq 0$. Indeed, since $n=2 k+2=2(k+1)$ is even, the right-hand sides $F^{i}(\theta)$ in (5.12) for this value of $n$ do not contain terms with wavenumber $\ell$.

\section{TWO APPROACHES FOR PROVING CONVERGENCE}

In $\S 5$ we determined an asymptotic series for the solution of (1.1)-(1.5), whose successive terms are defined as the solutions to the problems (5.11)-(5.12). As we said, a natural approach to a proof of convergence of the series suggests itself, namely the recursive estimation of the terms with the help of Lemma 5.1. This, of course, requires the estimation of the homogeneous terms $F^{i}$ at every step of the recursion and, from $\S 5$, we know that these functions can be expressed as sums

$$
\sum_{q} F_{q}^{i}
$$

where each $F_{q}^{i}$ is a product of the unknowns corresponding to previous stages of the recursive procedure and can therefore be estimated. Thus, at the $n$-th stage, the solution $\left(\sigma_{n}, p_{n}, f_{n}\right)$ can be written as

$$
\left(\sigma_{n}, p_{n}, f_{n}\right)=\sum_{q}\left(\sigma_{n}^{q}, p_{n}^{q}, f_{n}^{q}\right)
$$

where $\left(\sigma_{n}^{q}, p_{n}^{q}, f_{n}^{q}\right)$ solves (5.11)-(5.12) with $F^{i}$ replaced by $F_{q}^{i}$. Lemma 5.1 then would give, for instance,

$$
\left|\sigma_{n}\right|_{2+\alpha} \leq \sum_{q}\left|\sigma_{n}^{q}\right|_{2+\alpha} \leq C \sum_{q}\left(\left|F_{1}^{q}\right|_{2+\alpha}+\left|F_{2}^{q}\right|_{1+\alpha}+\left|F_{3}^{q}\right|_{\alpha}\right)
$$

and similar estimates for $\left|p_{n}\right|_{2+\alpha}$ and $\left|f_{n}\right|_{2+\alpha}$. Unfortunately, the recursive bounds on $\left(\sigma_{n}, p_{n}, f_{n}\right)$ that result from this approach do not imply the convergence of the series. Indeed, the need to expand $F^{i}$ as in (6.1) to derive a bound for use in (6.3) precludes this procedure from exploiting substantial cancellations that are present in (6.1). We illustrate this surprising phenomenon with a simple example. 
Consider the elliptic problem (a "scattering problem" at zero frequency) for a function $u=u(x, y)$ :

$$
\begin{gathered}
\Delta u=0 \quad \text { in } y>\varepsilon f(x), \\
u=H(x) \quad \text { on } y=\varepsilon f(x)
\end{gathered}
$$

where $f(x), H(x)$ are analytic functions, say

$$
\begin{gathered}
H(x)=\sum_{s=-F}^{F} h_{s} e^{i s x}, \\
f(x)=2 \cos x=e^{i x}+e^{-i x} ;
\end{gathered}
$$

we seek a solution which is $2 \pi$-periodic in $x$. By extending the problem to complex values of $\varepsilon$ it was shown in 4 that there exists an analytic solution $u(x, y, \varepsilon)$ for $y \geq 0,|\varepsilon|$ small, which remains bounded as $y \rightarrow \infty$ (see also [3]). We can then write

$$
u=\sum_{n=0}^{\infty} \varepsilon^{n} u_{n}(x, y)
$$

where the series is convergent for small $|\varepsilon|$. Let us now proceed to determine the coefficients $u_{n}$, and to estimate them, by the method of $\S 5$. Expanding the relation

$$
u(x, \varepsilon f(x), \varepsilon)=H(x)
$$

about $\varepsilon=0$, by Taylor's series, we obtain

$$
\begin{gathered}
u_{n}(x, 0)+\sum_{k=0}^{n-1} \frac{f^{n-k}}{(n-k) !} \partial_{y}^{n-k} u_{k}(x, 0)=0, n \geq 1, \\
u_{0}(x, 0)=H(x) .
\end{gathered}
$$

Writing

$$
\begin{gathered}
\frac{f(x)^{k}}{k !}=\sum_{r=-k}^{k} c_{k, r} e^{i r x}, \\
u_{m}(x, y)=\sum_{r=-F-m}^{F+m} d_{m, r} e^{i r x-|r| y},
\end{gathered}
$$

we find that

$$
u_{0}(x, y)=\sum_{s=-F}^{F} h_{s} e^{i s x-|s| y}
$$

and

$$
\sum_{r=-F-n}^{F+n} d_{n, r} e^{i r x}+\sum_{k=0}^{n-1}\left(\sum_{s=-(n-k)}^{n-k} c_{n-k, s} e^{i s x}\right) \sum_{q=-(F+k)}^{F+k} d_{k, q}(-|q|)^{n-k} e^{i q x}
$$

so that

$$
d_{n, r}+\sum_{k=0}^{n-1} \sum_{q=-(F+n-1)}^{F+n-1} c_{n-k, r-q} d_{k, q}(-|q|)^{n-k}=0, n \geq 1
$$


This equation is analogous to a modal form of (5.11)-(5.12). Let us take, in particular, $r=F+n$ and set $D_{n}=d_{n, F+n}$. Note that if $c_{n-k, F+n-q} d_{k, q} \neq 0$, then $F+n-q \leq n-k$ so that $F+k \leq q$. Since also $q \leq k+F$, we have $q=k+F$ and

$$
c_{n-k, F+n-q}=c_{n-k, n-k}=\frac{1}{(n-k) !} \quad \text { by }(6.6),(6.8) \text {. }
$$

Thus (6.10) gives

$$
D_{n}=-\sum_{k=0}^{n-1} \frac{D_{k}}{(n-k) !}(-(k+F))^{n-k}
$$

whereas $D_{0}=h_{F}$.

Multiplying (6.11) by $x^{n}$ and summing over $n$, we get

$$
\sum_{k \geq 0} x^{k} D_{k} \sum_{n \geq k} \frac{x^{n-k}}{(n-k) !}(-(k+F))^{n-k}=h_{F},
$$

or

$$
\sum_{k \geq 0} x^{k} D_{k} e^{-(k+F) x}=h_{F} .
$$

Hence

$$
\sum_{k \geq 0} x^{k} D_{k} e^{-k x}=h_{F} e^{F x} .
$$

Setting $D(z)=\sum_{k \geq 0} D_{k} z^{k}$ it follows that

$$
D\left(x e^{-x}\right)=h_{F} e^{F x} .
$$

Now introduce (by the implicit function theorem) the analytic function $\lambda(\xi)$ for $|\xi|$ small by

$$
\lambda\left(x e^{-x}\right)=x .
$$

Then we can rewrite (6.12) in the form

$$
D(y)=h_{F} e^{F \lambda(y)}, \quad|y| \text { small, }
$$

and since the right-hand side is analytic, we deduce that

$$
\left|D_{n}\right| \leq C_{0} H^{n} \text { for } n \geq 0
$$

where $C_{0}, H$ are positive constants. This estimate is of course also a consequence of the analyticity result in [4] obtained by working with complex $\epsilon$.

On the other hand, the analog of (6.3) in this case corresponds to the majorizing recursion

$$
\Delta_{n}=\sum_{k=0}^{n-1} \frac{\Delta_{k}}{(n-k) !}(k+F)^{n-k}
$$

obtained from (6.11) by taking absolute values and using the triangle inequality. We shall prove

$$
\limsup _{k \rightarrow \infty}\left|\Delta_{k}\right|^{1 / k}=\infty
$$


this will show that the estimate (6.13) (implied by analyticity) cannot be obtained in this manner. We take for simplicity $F=1$. Then, for any $M>1$,

$$
\Delta_{n M} \geq \Delta_{n(M-1)} \frac{(n(M-1)+1)^{n}}{n !} \geq \frac{M^{n}}{c^{n}} \Delta_{n(M-1)}
$$

where $c$ is a positive constant independent of $M, n$. By repeated application we get

$$
\Delta_{n M} \geq \frac{(M !)^{n}}{c^{n M}} \Delta_{n} .
$$

Then also

$$
\Delta_{M^{n}} \geq \frac{(M !)^{M^{n-1}}}{c^{M^{n}}} \Delta_{M^{n-1}} \geq \cdots \geq \frac{(M !)^{M^{n-1}+\cdots+M}}{c^{M^{n}+\cdots+M^{2}}} \Delta_{M},
$$

and this is true for any $M, n$. For any $c_{1}>c$ we can then choose $M$ large enough so that

$$
\Delta_{M^{n}} \geq \frac{(M !)^{M^{n-1}}}{\left(c_{1}\right)^{M^{n}}} \Delta_{M}
$$

Consequently,

$$
\left(\Delta_{M^{n}}\right)^{1 / M^{n}} \geq \frac{(M !)^{1 / M}}{c_{1}}\left(\Delta_{M}\right)^{1 / M^{n}} \geq \frac{M}{c_{2}} \text { if } n \rightarrow \infty,
$$

for any constant $c_{2}, c_{2}>e c_{1}$. Since $M$ is arbitrary, (6.15) follows.

The above example indicates that a proof of analyticity based on the recursive relations (6.7) must be extremely delicate, for one must take into account subtle cancellations that should occur in (6.10). The same phenomenon is expected of course of the much more complicated free boundary problem (1.1)-(1.5). We shall therefore use another approach. Let us describe it using again the simple example (6.4), (6.5).

We make a change of variables

$$
z=y-\varepsilon f(x)
$$

which flattens the boundary, and introduce the function

$$
v(x, z, \varepsilon)=u(x, y, \varepsilon) .
$$

Then $v$ satisfies the elliptic equation

$$
\begin{gathered}
\mathcal{L}_{\varepsilon} v \equiv \frac{\partial^{2} v}{\partial x^{2}}+\frac{\partial^{2} v}{\partial z^{2}}-2 \varepsilon f^{\prime} \frac{\partial^{2} v}{\partial x \partial z}+\varepsilon^{2}\left(f^{\prime}\right)^{2} \frac{\partial^{2} v}{\partial z^{2}}-\varepsilon f^{\prime \prime} \frac{\partial v}{\partial z}=0 \quad \text { in } z>0, \\
v(x, 0, \varepsilon)=H(x) .
\end{gathered}
$$

We can now determine a formal series

$$
v(x, z, \varepsilon)=\sum_{n=0}^{\infty} \varepsilon^{n} v_{n}(x, z)
$$

by applying $\left.D_{\varepsilon}^{n}\right|_{\varepsilon=0}$ to (6.18) successively. The function $\left.v_{n} \equiv \frac{1}{n !} D_{\varepsilon}^{n} v\right|_{\varepsilon=0}$ solves a system

$$
\begin{gathered}
\mathcal{L}_{0} v_{n}=F_{n} \quad \text { in } z>0, \\
v_{n}=0 \quad \text { if } \quad n \geq 1, \quad v_{0}=H(x) \quad \text { on } \quad z=0
\end{gathered}
$$


where $F_{n}$ depends on the $v_{m}$ with $m<n$. Using elliptic estimates we may then try to prove by induction on $n$ that

$$
\left.|| D_{x}^{k} D_{\varepsilon}^{n} v\right|_{\varepsilon=0} \| \leq C_{0} A^{k} C^{n}(n+k) ! \quad \forall k \geq 0
$$

where $C_{0}, A, C$ are some positive constants and the norm \| $\|$ is appropriately defined. Once (6.23) has been established for all $n$, we can then use the differential equation for $v$ to estimate also all the $z$-derivatives:

$$
\left.|| D_{z}^{j} D_{x}^{k} D_{\varepsilon}^{n} v\right|_{\varepsilon=0} \| \leq C_{0} B^{j} A^{k} C^{n}(n+k+j) !,
$$

and this will establish the convergence in (6.20) and the analyticity of $v(x, z, \varepsilon)$ in $(x, z, \varepsilon)$ for $-\infty<x<\infty, z \geq 0$ and $|\varepsilon|$ small.

This approach was used in 9 to prove analyticity up to the boundary for elliptic equations with boundary which is independent of $\varepsilon$. As we shall see in the following sections, this approach can be applied to the free boundary problem to establish analyticity of the solution $\sigma, p, f$ in the new variables; once this has been done, we can go back to the original variables and deduce the analyticity of $\sigma, p, f$ in the original variables. The "subtle cancellations" alluded to above in connection with the first approach to proving analyticity has thus been accomplished by the change to the new variables. Although we shall not be needing Lemma 5.1 in this second approach, some of the arguments used in the proof of the lemma will be useful.

\section{Change of variables}

The new approach, to be used throughout the rest of the paper, is based on transforming the free boundary problem (1.1)-(1.5) to a problem in the disc $\{r<1\}$ by means of the change of variable

$$
r=\frac{r^{\prime}}{R_{0}+f(\theta, \varepsilon)}
$$

of course $f(\theta, \varepsilon)$ is unknown. We define new functions $\widehat{\sigma}(r, \theta, \varepsilon), \widehat{p}(r, \theta, \varepsilon)$ by

$$
\widehat{\sigma}(r, \theta, \varepsilon)=\sigma\left(r^{\prime}, \theta, \varepsilon\right), \quad \widehat{p}(r, \theta, \varepsilon)=p\left(r^{\prime}, \theta, \varepsilon\right) .
$$

Then $\widehat{\sigma}, \widehat{p}$ satisfy the following system:

$$
\begin{gathered}
\mathcal{L}(D) \widehat{\sigma}-\left(R_{0}+f\right)^{2} \widehat{\sigma}=0 \quad \text { if } r<1, \\
\mathcal{L}(D) \widehat{p}+\left(R_{0}+f\right)^{2} \mu(\widehat{\sigma}-\tilde{\sigma})=0 \quad \text { if } r<1, \\
\widehat{\sigma}=\bar{\sigma} \quad \text { on } \quad r=1, \\
\left(R_{0}+f\right) \frac{\partial \widehat{p}}{\partial r}+\frac{\left(f_{\theta}\right)^{2}}{\left(R_{0}+f\right)} \frac{\partial \widehat{p}}{\partial r}-f_{\theta} \frac{\partial \widehat{p}}{\partial \theta}=0 \quad \text { on } r=1, \\
\left(\left(R_{0}+f\right)^{2}+f^{\prime 2}\right)^{3 / 2} \widehat{p}=\gamma\left[\left(R_{0}+f\right)^{2}+2\left(f_{\theta}\right)^{2}-\left(R_{0}+f\right) f_{\theta \theta}\right] \quad \text { on } \quad r=1,
\end{gathered}
$$

where

$$
\begin{aligned}
\mathcal{L}(D) & =\frac{\partial^{2}}{\partial r^{2}}+\frac{1}{r} \frac{\partial}{\partial r}+\frac{1}{r^{2}} \frac{\partial^{2}}{\partial \theta^{2}}-\frac{2 f_{\theta}}{\left(R_{0}+f\right)} \frac{1}{r} \frac{\partial^{2}}{\partial \theta \partial r} \\
& -\frac{f_{\theta \theta}}{\left(R_{0}+f\right)} \frac{1}{r} \frac{\partial}{\partial r}+2 \frac{\left(f_{\theta}\right)^{2}}{\left(R_{0}+f\right)^{2}} \frac{1}{r} \frac{\partial}{\partial r}+\frac{\left(f_{\theta}\right)^{2}}{\left(R_{0}+f\right)^{2}} \frac{\partial^{2}}{\partial r^{2}}
\end{aligned}
$$

Our approach to establishing branches of analytic solutions is to apply $D_{\varepsilon}^{n}(n=$ $1,2, \cdots)$ to the system $(7.3)-(7.7)$ at $\varepsilon=0$ in order to get recursive relations for $D_{\varepsilon}^{n} \widehat{\sigma}, D_{\varepsilon}^{n} \widehat{p}, D_{\varepsilon}^{n} f, \gamma_{n-1}($ at $\varepsilon=0)$ and then use a lemma analogous to Lemma 5.1 to estimate these derivatives. The right-hand sides that we shall obtain (and which 
are assumed known by the inductive assumption) are much simpler than the righthand sides that we had to deal with in $\S 5$; this is a critical fact, which will enable us to derive estimates on the derivatives that imply convergence and analyticity.

Taking $\varepsilon=0$ in the above system we get

$$
\begin{aligned}
& \widehat{\sigma}=S_{0}(r)=\bar{\sigma} \frac{I_{0}\left(R_{0} r\right)}{I_{0}\left(R_{0}\right)} \\
& \widehat{p}=P_{0}(r)=-\mu S_{0}(r)+A+\frac{\mu \tilde{\sigma}}{4} R_{0}^{2} r^{2}, P_{0}(1)=\frac{\gamma_{0}}{R_{0}}
\end{aligned}
$$

where $\gamma_{0}$ is the value of $\gamma$ at $\varepsilon=0$. If we linearize $(7.2)-(7.7)$ about $\left(S_{0}, P_{0}\right)$ by taking

$$
\widehat{\sigma}=S_{0}+\varepsilon S_{1}, \quad \widehat{p}=P_{0}+\varepsilon P_{1}
$$

where $r=1+\varepsilon \cos \ell \theta$ is the free boundary, we find that $\left(S_{1}, P_{1}\right)$ is a nontrivial solution if and only if $\gamma_{0}$ is given by (4.13). Indeed, if

$$
\begin{aligned}
& S_{1}=\left.\frac{d}{d \varepsilon}\right|_{\varepsilon=0}\left[\sigma_{0}\left(r\left(R_{0}+\varepsilon \cos \ell \theta\right)\right)+\varepsilon \sigma_{1}\left(r\left(R_{0}+\varepsilon \cos \ell \theta\right), \theta\right)\right], \\
& P_{1}=\left.\frac{d}{d \varepsilon}\right|_{\varepsilon=0}\left[p_{0}\left(r\left(R_{0}+\varepsilon \cos \ell \theta\right)\right)+\varepsilon p_{1}\left(r\left(R_{0}+\varepsilon \cos \ell \theta\right), \theta\right]\right.
\end{aligned}
$$

and $\gamma_{0}$ is as in $(4.13)$, then $\left(S_{1}, P_{1}\right)$ is a solution of the linearized problem. Conversely, if $\left(S_{1}, P_{1}\right)$ is a solution of the linearized problem corresponding to $f_{1}=$ $\cos \ell \theta$ and some $\gamma=\gamma^{\prime}$, then

$$
\begin{gathered}
\sigma_{1}=\left.\frac{d}{d \varepsilon}\right|_{\varepsilon=0}\left[S_{0}\left(\frac{r}{R_{0}+\varepsilon \cos \ell \theta}\right)+\varepsilon S_{1}\left(\frac{r}{R_{0}+\varepsilon \cos \ell \theta}, \theta\right)\right], \\
p_{1}=\left.\frac{d}{d \varepsilon}\right|_{\varepsilon=0}\left[P_{0}\left(\frac{r}{R_{0}+\varepsilon \cos \ell \theta}\right)+\varepsilon P_{1}\left(\frac{r}{R_{0}+\varepsilon \cos \ell \theta}, \theta\right)\right]
\end{gathered}
$$

form a solution of the linearized problem constructed in $\S 4$ and, consequently, by uniqueness, $\gamma^{\prime}$ must coincide with $\gamma_{0}$ as defined in (4.13).

We intend to prove that

$$
\begin{gathered}
\widehat{\sigma}(r, \theta, \varepsilon)=S_{0}(r)+\sum_{n=1}^{\infty} \varepsilon^{n} S_{n}(r, \theta), \\
\widehat{p}(r, \theta, \varepsilon)=P_{0}(r)+\sum_{n=1}^{\infty} \varepsilon^{n} P_{n}(r, \theta), \\
f(\theta, \varepsilon)=\sum_{n=1}^{\infty} \varepsilon^{n} f_{n}(\theta), \\
\gamma=\gamma_{0}+\gamma_{2} \varepsilon^{2}+\gamma_{4} \varepsilon^{n}+\cdots\left(\text { recall that } \gamma_{2 k+1}=0 ;\right. \text { see Remark 5.1) }
\end{gathered}
$$

where the series converge for $r \leq 1+\delta_{0},|\varepsilon| \leq \varepsilon_{0}$ and define analytic functions in $(x, y, \varepsilon)$ in the set $\left\{r \leq 1+\delta_{0}\right.$ and $\left.|\varepsilon| \leq \varepsilon_{0}\right\}$, for some $\varepsilon_{0}>0, \delta_{0}>0$.

To do this we shall first derive recursive formulas for

$$
S_{m}=\left.D_{\varepsilon}^{m} \widehat{\sigma}\right|_{\varepsilon=0}, P_{m}=\left.D_{\varepsilon}^{m} \widehat{p}\right|_{\varepsilon=0}, \wedge_{m}=\left.D_{\varepsilon}^{m} f\right|_{\varepsilon}=0:
$$

we assume that

$$
S_{m}, P_{m}-\gamma_{m} / R_{0}, \wedge_{m} \text { and } \gamma_{m-1}
$$

have already been determined for all $m<n$, and we shall proceed to determine these for $m=n$. 
To this end we differentiate the system (7.3)-(7.7) $n$ times with respect to $\varepsilon$ and set $\varepsilon=0$. Using the relations

$$
\frac{\partial S_{0}}{\partial \theta}=\frac{\partial P_{0}}{\partial \theta}=0, f_{\theta}=0 \text { at } \varepsilon=0, P_{0}(1)=\gamma_{0} / R_{0}
$$

we find that

$$
\begin{gathered}
\Delta S_{n}-\frac{\wedge_{n}, \theta \theta}{R_{0}} \frac{1}{r} \frac{\partial S_{0}}{\partial r}-2 R_{0} S_{0} \wedge_{n}-R_{0}^{2} S_{n}=F_{n}^{1}, r<1, \\
\Delta P_{n}-\frac{\wedge_{n}, \theta \theta}{R_{0}} \frac{1}{r} \frac{\partial P_{0}}{\partial r}+2 R_{0} \mu\left(S_{0}-\tilde{\sigma}\right) \wedge_{n}+\mu R_{0}^{2} S_{n}=F_{n}^{2}, r<1, \\
S_{n}=0 \quad \text { on } \quad r=1, \\
\frac{\partial P_{n}}{\partial r}=F_{n}^{3} \quad \text { on } \quad r=1, \\
P_{n}+\frac{\gamma_{0}}{R_{0}^{2}}\left(\wedge_{n}+\wedge_{n, \theta \theta}\right)=\frac{\gamma_{n-1}}{R_{0}^{2}}\left(\ell^{2}-1\right) \cos \ell \theta+\frac{\gamma_{n}}{R_{0}}+F_{n}^{4} \quad \text { on } r=1
\end{gathered}
$$

where the $F_{n}^{j}$ depend only on the functions (7.14) for $m<n$.

Note that the term $\left(\gamma_{n-1} / R_{0}\right)\left(\ell^{2}-1\right) \cos \ell \theta$ appears on the right-hand side of (7.19) in precisely the same way that it appeared in the third equation of (5.12); $F_{n}^{4}$ includes the term $P_{n-1}-\gamma_{n-1} / R_{0}$.

In the next section we prove an analog of Lemma 5.1 for the system (7.15)(7.19), but with Sobolev norms instead of Hölder norms. This lemma will enable us, in $\S 10$, to prove convergence and joint analyticity (in $(x, y)$ and $\varepsilon$ ) of the series (7.9)-(7.12).

\section{A fundamental Lemma}

Set $B=\{r<1\}, \partial B=\{r=1\}$. We shall need the following calculus type lemma:

Lemma 8.1. Let $u$ be any function in $H^{2}(B)$ which is even in $\theta$ and satisfies

$$
\begin{gathered}
\int_{0}^{2 \pi} u \cos \theta d \theta=0, \\
\left.u\right|_{\partial B}=0 .
\end{gathered}
$$

Then

$$
\begin{gathered}
\frac{1}{C}\|u\|_{H^{2}(B)} \leq\|u\|_{L^{2}(B)}+\left\|\frac{1}{r} \frac{\partial u}{\partial r}\right\|_{L^{2}(B)}+\left\|\frac{1}{r^{2}} \frac{\partial u}{\partial \theta}\right\|_{L^{2}(B)} \\
+\left\|\frac{1}{r^{2}} \frac{\partial^{2} u}{\partial \theta^{2}}\right\|_{L^{2}(B)}+\left\|\frac{1}{r} \frac{\partial^{2} u}{\partial r \partial \theta}\right\|_{L^{2}(B)}+\left\|\frac{\partial^{2} u}{\partial r^{2}}\right\|_{L^{2}(B)} \leq C\|u\|_{H^{2}(B)}
\end{gathered}
$$

where $C$ is a universal constant.

Proof. Setting $f=\Delta u$ we can write

$$
\begin{gathered}
u=\sum_{n \neq 1} u_{n}(r) \cos n \theta, \\
f=\sum_{n \neq 1} f_{n}(r) \cos n \theta
\end{gathered}
$$

where

$$
\frac{\partial^{2} u_{n}}{\partial r^{2}}+\frac{1}{r} \frac{\partial u_{n}}{\partial r}-\frac{n^{2}}{r^{2}} u_{n}=f_{n}(r), \quad u_{n}(1)=0
$$


It follows that

$$
u_{n}(r)=r^{n} \int_{1}^{r} \frac{d \rho}{\rho^{2 n+1}} \int_{0}^{\rho} \tau^{n+1} f_{n}(\tau) d \tau
$$

Consider first the case $n \geq 2$. Changing the order of integration we get

$$
\begin{aligned}
u_{n}(r) & =-r^{n} \int_{0}^{1} \tau^{n+1} f_{n}(\tau) d \tau \int_{\max (\tau, r)}^{1} \rho^{-2 n-1} d \rho \\
& =-r^{n} \int_{0}^{r} \tau^{n+1} f_{n}(\tau) \frac{\left(1-r^{-2 n}\right)}{(-2 n)} d \tau-r^{n} \int_{r}^{1} \tau^{n+1} f_{n}(\tau) \frac{\left(1-\tau^{-2 n}\right)}{(-2 n)} d \tau \\
& =\frac{r^{n}}{2 n} \int_{0}^{1} \tau^{n+1} f_{n}(\tau) d \tau-\int_{0}^{r} \frac{1}{2 n} r^{-n} \tau^{n+1} f_{n}(\tau) d \tau-\int_{r}^{1} \frac{r^{n}}{2 n} \tau^{-n+1} f_{n}(\tau) d \tau \\
& =\frac{1}{2 n}\left(I_{1}-I_{2}-I_{3}\right) .
\end{aligned}
$$

We have

$$
\begin{aligned}
\left(\int_{0}^{1}\left(\frac{I_{2}}{r^{2}}\right)^{2} r d r\right)^{1 / 2} & =\left(\int_{0}^{1}\left(\int_{0}^{r} r^{-n} \tau^{n+1} f_{n}(\tau) d \tau\right)^{2} \frac{1}{r^{4}} r d r\right)^{1 / 2} \\
& =\left(\int_{0}^{1}\left(\int_{0}^{1} u^{n+1} f_{n}(r u) d u\right)^{2} r d r\right)^{1 / 2} \quad(\tau=r u) \\
& \leq \int_{0}^{1} u^{n+1}\left(\int_{0}^{1} r\left(f_{n}(r u)\right)^{2} d r\right)^{1 / 2} d u
\end{aligned}
$$

by the generalized Minkowski inequality. Substituting $r=\tau / u$ in the inner integral, we get

$$
\left(\int_{0}^{1}\left(\frac{I_{2}}{r^{2}}\right)^{2} r d r\right)^{1 / 2} \leq \int_{0}^{1} u^{n}\left(\int_{0}^{u} f_{n}^{2}(\tau) \tau d \tau\right)^{1 / 2} d u \leq \frac{1}{n+1}\left(\int_{0}^{1} f_{n}^{2}(\tau) \tau d \tau\right)^{1 / 2} .
$$

Similarly,

$$
\begin{aligned}
\left(\int_{0}^{1}\left(\frac{I_{3}}{r^{2}}\right)^{2} r d r\right)^{1 / 2} & =\left(\int_{0}^{1}\left(\int_{r}^{1} r^{n} \tau^{-n+1} f_{n}(\tau) d \tau\right)^{2} \frac{1}{r^{4}} r d r\right)^{1 / 2} \\
& =\left(\int_{0}^{1}\left(\int_{r}^{1} u^{n-1} f_{n}\left(\frac{r}{u}\right) \frac{d u}{u^{2}}\right)^{2} r d r\right)^{1 / 2} \quad\left(\tau=\frac{r}{u}\right) \\
& \leq \int_{0}^{1} u^{n-3}\left(\int_{0}^{u}\left(f_{n}\left(\frac{r}{u}\right)\right)^{2} r d r\right)^{1 / 2} d u \\
& \leq \int_{0}^{1} u^{n-3}\left(\int_{0}^{1} u^{2} f_{n}(\tau)^{2} \tau d \tau\right)^{1 / 2} d u \quad(r=\tau u) \\
& \leq\left(\int_{0}^{1} u^{n-2} d u\right)\left(\int_{0}^{1} f_{n}(\tau)^{2} \tau d \tau\right)^{1 / 2} \\
& =\frac{1}{n-1}\left(\int_{0}^{1} f_{n}(\tau)^{2} \tau d \tau\right)^{1 / 2} .
\end{aligned}
$$

Since $I_{1} \leq I_{2}+I_{3}$, we conclude that

$$
\left[\int_{0}^{1} \frac{1}{r^{4}}\left(u_{n}(r)\right)^{2} r d r\right]^{1 / 2} \leq \frac{4}{2(n-1) n}\left[\int_{0}^{1} f_{n}(\tau)^{2} \tau d \tau\right]^{1 / 2} .
$$


Consider next the case $n=0$. We then have

$$
u_{0}(\tau)=\int_{0}^{r} \tau f_{0}(\tau) d \tau \cdot \log r+\int_{r}^{1} \tau f_{0}(\tau) \log \tau d \tau
$$

so that

$$
\frac{\partial u_{0}}{\partial r}=\frac{1}{r} \int_{0}^{r} \tau f_{0}(\tau) d \tau
$$

We can now proceed to estimate the $L^{2}$ norm of $\frac{1}{r} \frac{\partial u}{\partial r}$. Note first that

$$
\frac{1}{r} \frac{\partial u_{n}}{\partial r}=n \frac{u_{n}}{r^{2}}+r^{-n-2} \int_{0}^{r} \tau^{n+1} f_{n}(\tau) d \tau=n \frac{u_{n}}{r^{2}}+\frac{I_{2}(r)}{r^{2}} .
$$

Hence, by (8.6) and (8.5),

It easily follows that

$$
\left(\int_{0}^{1}\left(\frac{1}{r} \frac{\partial u_{n}}{\partial r}\right)^{2} r d r\right)^{1 / 2} \leq \frac{C}{n}\left(\int_{0}^{1} f_{n}(\tau)^{2} \tau d \tau\right)^{1 / 2} .
$$

$$
\left\|\frac{1}{r} \frac{\partial u}{\partial r}\right\|_{L^{2}(R)}+\left\|\frac{1}{r} \frac{\partial^{2} u}{\partial r \partial \theta}\right\|_{L^{2}(B)} \leq C\|f\|_{L^{2}(B)} .
$$

From (8.6) we also deduce that

$$
\left\|\frac{1}{r^{2}} \frac{\partial u}{\partial \theta}\right\|_{L^{2}(B)}+\left\|\frac{1}{r^{2}} \frac{\partial^{2} u}{\partial \theta^{2}}\right\|_{L^{2}(B)} \leq C\|f\|_{L^{2}(R)} .
$$

Finally,

$$
\left\|\frac{\partial^{2} u}{\partial r^{2}}\right\|_{L^{2}(B)}=\left\|\Delta u-\frac{2}{r} \frac{\partial u}{\partial r}-\frac{1}{r^{2}} \frac{\partial^{2} u}{\partial \theta^{2}}\right\|_{L^{2}(B)} \leq C\|f\|_{L^{2}(B)} .
$$

Remark 8.1. The condition (8.1) cannot be dropped. Indeed, the function $u=$ $\left(r-r^{3}\right) \cos \theta$ satisfies (8.2) but not (8.1), and $\frac{1}{r} u_{r}$ is not in $L^{2}(B)$.

We now state a fundamental lemma, an analog to Lemma 5.1, for the system (7.15)-(7.19).

Lemma 8.2. Consider the problem

$$
\begin{gathered}
\Delta S-\frac{1}{R_{0}} \wedge_{\theta \theta} \frac{1}{r} \frac{\partial S_{0}}{\partial r}-2 R_{0} S_{0} \wedge-R_{0}^{2} S=F^{1} \quad \text { in } B \\
\Delta P-\frac{1}{R_{0}} \wedge_{\theta \theta} \frac{1}{r} \frac{\partial P_{0}}{\partial r}+2 R_{0} \mu\left(S_{0}-\tilde{\sigma}\right) \wedge+\mu R_{0}^{2} S=F^{2} \quad \text { in } B \\
S=0 \quad \text { on } \quad \partial B \\
\frac{\partial P}{\partial r}=F^{3} \quad \text { on } \quad \partial B \\
P+\frac{\gamma_{0}}{R_{0}^{2}}\left(\wedge+\wedge_{\theta \theta}\right)=\frac{\gamma}{R_{0}^{2}}\left(\ell^{2}-1\right) \cos \ell \theta+F^{4} \quad \text { on } \quad \partial B
\end{gathered}
$$

where $\ell \geq 2, F^{1} \in L^{2}(B), F^{2} \in L^{2}(B), F^{3} \in H^{1 / 2}(\partial B), F^{4} \in H^{3 / 2}(\partial B), F^{j}$ is even in $\theta$, and

$$
\int_{0}^{2 \pi} F^{j} \cos \theta d \theta=0 \quad(1 \leq j \leq 4) .
$$

Set

$$
\|F\|=\left\|F^{1}\right\|_{L^{2}(B)}+\left\|F^{2}\right\|_{L^{2}(B)}+\left\|F^{3}\right\|_{H^{1 / 2}(\partial B)}+\left\|F^{4}\right\|_{L^{2}(\partial B)} .
$$


Then there exists a unique solution $(S, P, \wedge, \gamma)$ of $(8.9)-(8.13)$ with $S, P$ in $H^{2}(B), \wedge$ in $H^{2}(\partial B)$ such that $S, P, \wedge$ are even in $\theta$ and

$$
\begin{gathered}
\int_{0}^{2 \pi}\left(\begin{array}{l}
S \\
P
\end{array}\right) \cos \theta d \theta=0 \\
\int_{0}^{2 \pi} \wedge(\theta) \cos m \theta d \theta=0 \text { for } m=1, \ell ;
\end{gathered}
$$

furthermore,

$$
|\gamma| \leq C\left\{\left[\int_{0}^{1}\left|\int_{0}^{2 \pi} d \theta F^{1} \cos \ell \theta\right|^{2} r d r\right]^{1 / 2}+\left[\int_{0}^{1}\left|\int_{0}^{2 \pi} d \theta F^{2} \cos \ell \theta\right|^{2} r d r\right]^{1 / 2}\right.
$$

$$
\begin{aligned}
\left\|\frac{1}{r} \frac{\partial P}{\partial r}\right\|_{L^{2}(B)} & +\left\|\frac{1}{r^{2}} \frac{\partial P}{\partial \theta}\right\|_{L^{2}(B)}+\left\|\frac{1}{r^{2}} \frac{\partial^{2} P}{\partial \theta^{2}}\right\|_{L^{2}(B)} \\
& +\left\|\frac{1}{r} \frac{\partial^{2} P}{\partial r \partial \theta}\right\|_{L^{2}(B)}+\left\|\frac{\partial^{2} P}{\partial r^{2}}\right\|_{L^{2}(B)} \leq C\left(\|F\|+\left\|F^{4}\right\|_{H^{3 / 2}(\partial B)}\right)
\end{aligned}
$$

and

$$
\|\wedge\|_{H^{7 / 2}(\partial B)} \leq C\left(\|F\|+\left\|F^{4}\right\|_{H^{3 / 2}(\partial B)}\right) ;
$$

the constant $C$ in the above estimates is independent of the $F^{i}$.

Proof. The assumptions of the lemma imply that

$$
F^{j}=\sum_{m \neq 1} F_{m}^{j} \cos m \theta
$$

and that any solution of (8.9)-(8.13) can be written in the form

$$
\begin{aligned}
S & =\sum_{m \neq 1} T_{m}(r) \cos m \theta, \\
P & =\sum_{m \neq 1} Q_{m}(r) \cos m \theta, \\
\wedge & =\sum_{m \neq 1} \Gamma_{m} \cos m \theta
\end{aligned}
$$

we do not as yet impose the orthogonality condition $\int_{0}^{2 \pi} \wedge(\theta) \cos \ell \theta d \theta=0$. 
We then obtain the system of differential equations

$$
\begin{aligned}
& \frac{\partial^{2}}{\partial r^{2}} T_{m}+\frac{1}{r} \frac{\partial}{\partial r} T_{m}-\frac{m^{2}}{r^{2}} T_{m}-R_{0}^{2} T_{m} \\
& \quad+\left(\frac{m^{2}}{r R_{0}} \bar{\sigma} \frac{R_{0} I_{1}\left(R_{0} r\right)}{I_{0}\left(R_{0}\right)}-2 R_{0} \bar{\sigma} \frac{I_{0}\left(R_{0} r\right)}{I_{0}\left(R_{0}\right)}\right) \Gamma_{m}=F_{m}^{1} \text { in } B
\end{aligned}
$$

$$
\begin{aligned}
\frac{\partial^{2}}{\partial r^{2}} Q_{m}+ & \frac{1}{r} \frac{\partial}{\partial r} Q_{m}-\frac{m^{2}}{r^{2}} Q_{m}+\mu R_{0}^{2} T_{m} \\
+\left\{-\frac{m^{2}}{r R_{0}} \mu \bar{\sigma} \frac{R_{0} I_{1}\left(R_{0} r\right)}{I_{0}\left(R_{0}\right)}+\right. & \frac{m^{2}}{r R_{0}} \frac{\mu \tilde{\sigma} R_{0}^{2} r}{2} \\
& \left.+2 R_{0} \mu \bar{\sigma} \frac{I_{0}\left(R_{0} r\right)}{I_{0}\left(R_{0}\right)}-2 R_{0} \mu \tilde{\sigma}\right\} \Gamma_{m}=F_{m}^{2} \text { in } B,
\end{aligned}
$$

with the boundary conditions

$$
\begin{aligned}
T_{m} & =0 \quad \text { on } \partial B \\
\frac{\partial Q_{m}}{\partial r} & =F_{m}^{3} \quad \text { on } \partial B \\
Q_{m}-\frac{\gamma_{0}}{R_{0}^{2}}\left(m^{2}-1\right) \Gamma_{m} & =\frac{\delta_{0}}{R_{0}^{2}}\left(\ell^{2}-1\right) \delta_{m \ell}+F_{m}^{4} \quad \text { on } \partial B .
\end{aligned}
$$

The function

$$
w=\mu T_{m}+Q_{m}
$$

satisfies

$$
\frac{\partial^{2} w}{\partial r^{2}}+\frac{1}{r} \frac{\partial w}{\partial r}-\frac{m^{2}}{r^{2}} w=M_{m} \Gamma_{m}+\left(\mu F_{m}^{1}+F_{m}^{2}\right)
$$

where $M_{m}=-\frac{1}{2} \mu \tilde{\sigma}\left(m^{2}-4\right) R_{0}$. The general solution of this equation which is regular in $B$ is

$$
\mu \frac{\tilde{\sigma}}{2} R_{0} r^{2} \Gamma_{m}+K_{m} r^{m}+r^{m} \int_{1}^{r} \frac{d s}{s^{2 m+1}} \int_{0}^{s} \tau^{m+1}\left(\mu F_{m}^{1}+F_{m}^{2}\right) d \tau
$$

where $K_{m}$ is an arbitrary constant. Hence

$$
\begin{aligned}
Q_{m}(r)= & -\mu T_{m}(r)+\mu \frac{\tilde{\sigma}}{2} R_{0} r^{2} \Gamma_{m}+K_{m} r^{m} \\
& +r^{m} \int_{1}^{r} \frac{d s}{s^{2 m+1}} \int_{0}^{s} \tau^{m+1}\left(\mu F_{m}^{1}(\tau)+F_{m}^{2}(\tau)\right) d \tau .
\end{aligned}
$$

Next we calculate $T_{m}(r)$. Setting

$$
g_{m}(r)=\left[m^{2} I_{1}\left(R_{0} r\right)-2 R_{0} r I_{0}\left(R_{0} r\right)\right] \frac{\bar{\sigma}}{I_{0}\left(R_{0}\right)}
$$

and trying a solution of the form

$$
T_{m}(r)=I_{m}\left(R_{0} r\right) v(r),
$$

we get

$$
\frac{1}{r I_{m}\left(R_{0} r\right)}\left(r\left(I_{m}\left(R_{0} r\right)\right)^{2} v^{\prime}\right)^{\prime}=-\frac{g_{m}(r)}{r} \Gamma_{m}+F_{m}^{1}
$$


Hence

$$
\begin{aligned}
T_{m}(r)= & -I_{m}\left(R_{0} r\right) \int_{1}^{r} \frac{d s}{s I_{m}\left(R_{0} s\right)^{2}} \int_{0}^{s} I_{m}\left(R_{0} \tau\right) g_{m}(\tau) d \tau \cdot \Gamma_{m} \\
& +I_{m}\left(R_{0} r\right) \int_{1}^{r} \frac{d s}{s I_{m}\left(R_{0} s\right)^{2}} \int_{0}^{s} \tau I_{m}\left(R_{0} \tau\right) F_{m}^{1}(\tau) d \tau
\end{aligned}
$$

notice that $T_{m}(1)=0$. Thus it remains to choose the constants $K_{m}, \Gamma_{m}$ in such a way that the boundary conditions (8.29) and (8.30) are satisfied. Using (8.31) and (8.33), these conditions become

$$
A_{m}\left(\begin{array}{c}
\Gamma_{m} \\
K_{m}
\end{array}\right)=\left(\begin{array}{c}
\widetilde{F}_{m}^{1} \\
\widetilde{F}_{m}^{2}
\end{array}\right)
$$

where

$$
A_{m}=\left(\begin{array}{lc}
\mu \tilde{\sigma} R_{0}+\frac{\mu}{I_{m}\left(R_{0}\right)} \int_{0}^{1} I_{m}\left(R_{0} \tau\right) g_{m}(\tau) d \tau & m \\
\mu \frac{\tilde{\sigma}}{2} R_{0}-\frac{\gamma_{0}}{R_{0}^{2}}\left(m^{2}-1\right) & 1
\end{array}\right)
$$

and

$$
\begin{aligned}
& \widetilde{F}_{m}^{1}=\frac{\mu}{I_{m}\left(R_{0}\right)} \int_{0}^{1} \tau I_{m}\left(R_{0} \tau\right) F_{m}^{1}(\tau) d \tau-\int_{0}^{1} \tau^{m+1}\left(\mu F_{m}^{1}(\tau)+F_{m}^{2}(\tau)\right) d \tau+F_{m}^{3}, \\
& \widetilde{F}_{m}^{2}=\frac{\gamma}{R_{0}^{2}}\left(\ell^{2}-1\right) \delta_{m \ell}+F_{m}^{4} .
\end{aligned}
$$

From Lemma 4.3 we see that $\operatorname{det} A_{m} \neq 0$ if $m \neq \ell$ and therefore the system (8.34) has a unique solution if $m \neq \ell$ (note that $\widetilde{F}_{m}^{2}=F_{m}^{4}$ if $m \neq \ell$ ). On the other hand, if $m=\ell$, then $\operatorname{det} A_{\ell}=0, \delta_{m \ell}=1$ and a solution exists if and only if the first row of the augmented matrix is a multiple of the second row; the multiple is necessarily $\ell$. We thus get the consistency condition $\ell \widetilde{F}_{\ell}^{2}=\widetilde{F}_{\ell}^{1}$ or,

$$
\frac{\ell\left(\ell^{2}-1\right)}{R_{0}^{2}} \gamma=\widetilde{F}_{\ell}^{1}-\ell F_{\ell}^{4}
$$

This determines $\gamma$ uniquely, and also proves the estimate (8.21).

There is an infinite number of solutions $\left(T_{\ell}, Q_{\ell}, \Gamma_{\ell}\right)$, obtained from one solution by adding a multiple of $\cos \ell \theta$ to $\wedge$. We determine a unique solution by requiring that $\Gamma_{\ell}=0$, i.e.,

$$
\int_{0}^{2 \pi} \wedge(\theta) \cos \ell \theta d \theta=0
$$

We proceed to derive the estimates (8.18)-(8.20). By elliptic estimates (using (8.9) and (8.11))

$$
\|S\|_{H^{2}(B)} \leq C\left(\left\|F^{1}\right\|_{L^{2}(B)}+\|\wedge\|_{H^{2}(\theta B)}\right) .
$$

Similarly, from (8.10) and (8.12),

$$
\begin{aligned}
\|P\|_{H^{2}(B)} \leq C\left(\left\|F^{2}\right\|_{L^{2}(B)}+\left\|F^{3}\right\|_{H^{1 / 2}(\partial B)}+\|S\|_{L^{2}(B)}\right. \\
\left.+\|\wedge\|_{H^{2}(\partial B)}+\|P\|_{L^{2}(B)}\right) .
\end{aligned}
$$

From (8.13) and the trace theorem [2] we also have

$$
\|\wedge\|_{H^{2}(\partial B)} \leq C\left(\|P\|_{H^{1}(B)}+\left\|F^{4}\right\|_{L^{2}(\partial B)}+|\gamma|\right) .
$$


We claim that there is a constant $C_{0}$ independent of the $F^{i}$ such that

$$
\|P\|_{H^{1}(B)} \leq C_{0}\|F\| \text {. }
$$

Indeed, otherwise there is a sequence $\left(F_{j}^{1}, F_{j}^{2}, F_{j}^{3}, F_{j}^{4}\right) \equiv F_{j}$ and corresponding solutions $\left(S_{j}, P_{j}, \wedge_{j}, \gamma_{j}\right)$ such that

$$
1=\left\|P_{j}\right\|_{H^{1}(B)} \geq j\left\|F_{j}\right\| .
$$

From (8.21), (8.35)-(8.37) we deduce that for a subsequence, $S_{j} \rightarrow \widetilde{S}, P_{j} \rightarrow \widetilde{P}$ weakly in $H^{2}(B), \wedge_{j} \rightarrow \widetilde{\wedge}$ weakly in $H^{2}(B)$ and $\gamma_{j} \rightarrow \widetilde{\gamma}$, whereas $F_{j}^{k} \rightarrow 0(1 \leq k \leq$ $4)$ in the norm of $(8.15)$. It follows that $(\widetilde{S}, \widetilde{P}, \widetilde{\wedge}, \widetilde{\gamma})$ is a solution of the homogeneous system $(8.9)-(8.13)$ and, by uniqueness, $\widetilde{S} \equiv 0, \widetilde{P} \equiv 0$. But

a contradiction.

$$
\|\widetilde{P}\|_{H^{1}(B)}=\lim \left\|P_{j}\right\|_{H^{1}(B)}=1
$$

Having proved (8.38), we use it in (8.37) to conclude the proof of (8.20). Next, substituting (8.20) into (8.35), (8.36), the inequalities (8.18), (8.19) follow.

Observe that the estimate (8.22) follows from (8.18) and Lemma 8.1, and the estimate (8.24) follows from (8.13) and (8.19). Thus it remains to prove (8.23). Since $P(1, \theta) \not \equiv 0$ this estimate does not follow directly from Lemma 8.1. However, the part of $P$ which comes from the first and last terms on the right-hand side of (8.31) are estimated by (8.22) and Lemma 8.1. Thus it remains to estimate

$$
\begin{gathered}
\left\|\frac{1}{r} \frac{\partial \widetilde{P}}{\partial r}\right\|_{L^{2}(B)}, \quad\left\|\frac{1}{r} \frac{\partial^{2} \widetilde{P}}{\partial r \partial \theta}\right\|_{L^{2}(B)} \\
\left\|\frac{1}{r^{2}} \frac{\partial \widetilde{P}}{\partial \theta}\right\|_{L^{2}(B)}, \quad\left\|\frac{1}{r^{2}} \frac{\partial^{2} \widetilde{P}}{\partial \theta^{2}}\right\|_{L^{2}(B)}, \quad\left\|\frac{\partial^{2} \widetilde{P}}{\partial r^{2}}\right\|_{L^{2}(B)}
\end{gathered}
$$

where

$$
\widetilde{P}=\mu \frac{\tilde{\sigma}}{2} R_{0} r^{2} \sum_{m \neq 1} \Gamma_{m} \cos m \theta+\sum_{m \neq 1} K_{m} r^{m} \cos m \theta \equiv P_{1}+P_{2} .
$$

The estimates for $P_{1}$ follow immediately from the inequality

$$
\sum\left(m^{7 / 2} \Gamma_{m}\right)^{2} \leq C\|\wedge\|_{H^{7 / 2}(\partial B)}
$$

and (8.24).

Next from the second equation of (8.34) we get

$$
\left|K_{m}\right| \leq C m^{2}\left|\Gamma_{m}\right|+C \gamma \delta_{m l}+C\left|F_{m}^{4}\right|
$$

and therefore

$$
\left\|\frac{1}{r^{2}} \frac{\partial^{2} P_{2}}{\partial \theta^{2}}\right\|_{L^{2}(B)} \leq C\left(\sum_{m>1} m^{3}\left|K_{m}\right|^{2}\right) \leq C\left(\gamma+\|\wedge\|_{H^{7 / 2}(\partial B)}+\left\|F^{4}\right\|_{H^{3 / 2}(\partial B)}\right) .
$$

The remaining partial derivatives of $P_{2}$ which occur in (8.39) (with $\widetilde{P}$ replaced by $P_{2}$ ) are estimated in the same way.

We shall need, in addition to Lemma 8.2, its counterpart for odd functions of $\theta$.

Lemma 8.3. Consider the system (8.9)-(8.13) with $\cos \ell \theta$ replaced by $\sin \ell \theta$ in (8.13) and $F^{j}$ as in Lemma 8.2 but odd functions in $\theta$, satisfying

$$
\int_{0}^{2 \pi} F^{j} \sin \theta d \theta=0 \quad(1 \leq j \leq 4)
$$


instead of (8.14). Then there exists a unique solution $(S, P, \wedge, \gamma)$ with $S, P$ in $H^{2}(B), \wedge$ in $H^{2}(\partial B)$ such that $S, P, \wedge$ are odd in $\theta$ and

$$
\begin{gathered}
\int_{0}^{2 \pi}\left(\begin{array}{l}
S \\
P
\end{array}\right) \sin \theta d \theta=0, \\
\int_{0}^{2 \pi} \wedge(\theta) \sin m \theta d \theta=0 \text { for } m=1, \ell,
\end{gathered}
$$

and the estimates (8.18)-(8.24) hold with $\cos \ell \theta$, in (8.21), replaced by $\sin \ell \theta$.

\section{AN AUXiLiary LEMma}

We shall need the following known estimates [2]:

$$
\begin{gathered}
\|f\|_{L^{\infty}(\partial B)} \leq C\|f\|_{H^{s}(\partial B)}, \\
\|f g\|_{H^{1 / 2}(\partial B)} \leq C\|f\|_{H^{s}(\partial B)}\|g\|_{H^{1 / 2}(\partial B)} \text { and } \\
\|f g\|_{H^{s}(\partial B)} \leq C\|f\|_{H^{s}(\partial B)}\|g\|_{H^{s}(\partial B)},
\end{gathered}
$$

where $s>\frac{1}{2}$ and $C$ is a constant depending only on $s$. We shall also need the inequality

$$
\left(\begin{array}{l}
a \\
b
\end{array}\right)\left(\begin{array}{l}
c \\
d
\end{array}\right) \leq\left(\begin{array}{l}
a+c \\
b+d
\end{array}\right)
$$

which follows by induction on $a(a \geq b)$ using the relation

$$
\left(\begin{array}{l}
a \\
b
\end{array}\right)=\left(\begin{array}{l}
a-1 \\
b-1
\end{array}\right)+\left(\begin{array}{c}
a-1 \\
b
\end{array}\right)
$$

Lemma 9.1. Let

$$
F(x)=\sum_{n \geq 1} \alpha_{n} x^{n}, \quad \lambda(\theta, \varepsilon)=\sum_{n \geq 1} \lambda_{n}(\theta) \varepsilon^{n}
$$

be such that $\left|\alpha_{n}\right| \leq C^{n}$ and, for some $s>\frac{1}{2}$,

$$
\left\|D_{\theta}^{k} \lambda_{n}\right\|_{H^{s}(\partial B)} \leq C_{0} A^{k-2} H^{n-2} \frac{(k+n) !}{(k+n)^{3} n !} \quad \forall k, n
$$

where $C_{0}, A, H$ are positive constants, and $A>1, H>1$. Set

$$
F(\lambda(\theta, \varepsilon))=\sum_{n \geq 1} F_{n}(\theta) \varepsilon^{n} .
$$

Then

$$
\left\|D_{\theta}^{k} F_{n}(\theta)\right\|_{H^{s}(\partial B)} \leq C_{0} K A^{k-2} H^{n-2} \frac{(k+n) !}{(k+n)^{3} n !}
$$

for all $n, k$, provided $H \geq 2 C C_{0} S$, where $S$ is a universal constant and $K$ depends only on $C, C_{0}$.

The lemma is similar to one proved in [9]. In (9.3), (9.5) we use the following convention:

$$
A^{-j}=H^{-j}=1 \quad \text { if } \quad j \geq 0 .
$$


Proof. We shall abbreviate $\|\cdots\|_{H^{s}(\partial B)}$ by $\|\cdots\|_{s}$. Consider first the case $F(x)=$ $x^{m}$. We write

$$
\left(\sum_{n \geq 1} \lambda_{n}(\theta) \varepsilon^{n}\right)^{m}=\sum_{n \geq m} \varphi_{n}^{m}(\theta) \varepsilon^{n}
$$

and proceed to prove by induction on $m$ that

$$
\left\|D_{\theta}^{k} \varphi_{n}^{m}\right\|_{s} \leq S^{m-1} C_{0}^{m} A^{k-2} H^{n-1-m} \frac{(k+n) !}{(k+n)^{3} n !} .
$$

From (9.3) this holds for $m=1$. To go from $m$ to $m+1$ we shall make use of the relation

$$
\varphi_{n}^{m+1}(\theta)=\sum_{j=m}^{n-1} \varphi_{j}^{m}(\theta) \lambda_{n-j}(\theta) .
$$

Using (9.1) we see that

$$
\begin{aligned}
\left\|D_{\theta}^{k} \varphi_{n}^{m+1}\right\|_{s} \leq & \sum_{j=m}^{n-1} \sum_{l=0}^{k} \frac{k !}{l !(k-l) !}\left\|D_{\theta}^{l} \varphi_{j}^{m}\right\|_{s}\left\|D_{\theta}^{k-l} \lambda_{n-j}\right\|_{s} \\
\leq & \sum_{j=m}^{n-1} \sum_{l=0}^{k} \frac{k !}{l !(k-l) !} A^{l-2} H^{j-1-m} C_{0}^{m} S^{m-1} \frac{(j+l) !}{(j+l)^{3} j !} \\
& \times C_{0} A^{k-l-2} H^{n-j-2} \frac{(n-j+k-l) !}{(n-j+k-l)^{3}(n-j) !} .
\end{aligned}
$$

Using (9.2) we get the bound

$$
C_{0}^{m+1} S^{m} A^{k-2} H^{n-1-(m+1)} \frac{(k+n) !}{(k+n)^{3} n !}
$$

where

$$
S \geq \sum_{l=0}^{k} \sum_{j=1}^{n-1} \frac{(k+n)^{3}}{(j+l)^{3}(k+n-j-l)^{3}}
$$

is a universal constant. This completes the proof of (9.7).

Consider now the general case of $F(x)$, and write

$$
\begin{aligned}
& F(\lambda(\theta, \varepsilon))=\sum_{m \geq 1} \alpha_{m}(\lambda(\theta, \varepsilon))^{m} \\
& \quad=\sum_{m \geq 1} \alpha_{m} \sum_{n \geq m} \varphi_{n}^{m}(\theta) \varepsilon^{n}=\sum_{n \geq 1}\left(\sum_{m=1}^{n} \alpha_{m} \varphi_{n}^{m}(\theta)\right) \varepsilon^{n}
\end{aligned}
$$

so that, by $(9.4)$,

$$
F_{n}(\theta)=\sum_{m=1}^{n} \alpha_{m} \varphi_{n}^{m}(\theta)
$$


Using (9.7) we get

$$
\begin{aligned}
& \left\|D_{\theta}^{k} F_{n}\right\|_{s} \leq \sum_{m=1}^{n}\left|\alpha_{m}\right| A^{k-2} H^{n-1-m} C_{0}^{m} S^{m-1} \frac{(k+n) !}{(k+n)^{3} n !} \\
& \quad \leq \frac{(k+n) !}{(k+n)^{3} n !} A^{k-2}\left[H^{n-2} \sum_{m=1}^{n-1} \frac{\left|\alpha_{m}\right| C_{0}^{m} S^{m-1}}{H^{m-1}}+\left|\alpha_{n}\right| C_{0}^{n} S^{n-1}\right] \\
& \quad \leq \frac{(k+n) !}{(k+n)^{3} n !} A^{k-2} H^{n-2} C_{0} K
\end{aligned}
$$

provided $H \geq 2 C C_{0} S$, where $K$ depends only on $C_{0}, C$.

\section{Convergence}

In $\S \S 7$ and 8 we determined a formal solution (7.9)-(7.12) to the system (7.3)(7.7), showing that the system (7.15)-(7.19) has a unique solution (even in $\theta$ ) subject to the orthogonality conditions

$$
\int_{0}^{2 \pi} f_{j}(\theta) \cos \ell \theta d \theta=0 \quad(j \geq 2) .
$$

The modes that appear in (7.15)-(7.19) are $\cos m \ell \theta$ where $m$ is a nonnegative integer. Using Lemmas 8.2 and 8.3 we shall prove the following theorem.

Theorem 10.1. The series (7.9)-(7.12) are uniformly convergent for $\varepsilon^{\prime}<r \leq 1+$ $\delta_{0},|\varepsilon| \leq \varepsilon_{0}$ where $\delta_{0}$ and $\varepsilon_{0}$ are positive numbers and $\varepsilon^{\prime}$ is arbitrarily small positive number; furthermore, the functions $\widehat{\sigma}(r, \theta, \varepsilon), \widehat{p}(r, \theta, \varepsilon)$ are analytic in $(r, \theta, \varepsilon)$ for $0<r \leq 1+\delta_{0},|\varepsilon| \leq \varepsilon_{0}$ and the function $f(\theta, \varepsilon)$ is analytic in $(\theta, \varepsilon)$ for $0 \leq \theta \leq$ $2 \pi,|\varepsilon| \leq \varepsilon_{0}$.

The proof is given in this and the next section.

To prove the theorem we introduce the norm

$$
\begin{aligned}
\|u\|_{H_{r}^{2}(B)} & =\|u\|_{L^{2}(B)}+\left\|\frac{1}{r} \frac{\partial u}{\partial r}\right\|_{L^{2}(B)}+\left\|\frac{1}{r^{2}} \frac{\partial u}{\partial \theta}\right\|_{L^{2}(B)} \\
& +\left\|\frac{1}{r^{2}} \frac{\partial^{2} u}{\partial \theta^{2}}\right\|_{L^{2}(B)}+\left\|\frac{1}{r} \frac{\partial^{2} u}{\partial r \partial \theta}\right\|_{L^{2}(B)}+\left\|\frac{\partial^{2} u}{\partial r^{2}}\right\|_{L^{2}(B)}
\end{aligned}
$$

and derive the following estimates:

$$
\begin{gathered}
\left\|\partial_{\theta}^{j} S_{m}\right\|_{H_{r}^{2}(B)}+\left\|\partial_{\theta}^{j}\left(P_{m}-\frac{\gamma_{m}}{R_{0}}\right)\right\|_{H_{r}^{2}(B)} \leq C_{0} A^{j-2} H^{m-2} \frac{(j+m) !}{(j+m)^{3} m !}, \\
\left\|\partial_{\theta}^{j} f_{m}\right\|_{H^{7 / 2}(\partial B)} \leq C_{0} A^{j-2} H^{m-2} \frac{(j+m) !}{(j+m)^{3} m !}, \\
\left|\gamma_{m-1}\right| \leq C_{0} \frac{H^{m-2}}{m^{3}}
\end{gathered}
$$

for all $m \geq 0, j \geq 0, m+j>0$ in (10.2), (10.3) and $m \geq 1$ in (10.4).

The proof is by induction on $m$. We assume that (10.2)-(10.4) hold for all $m<n$, and proceed to prove these inequalities for $m=n$. For simplicity we take $n \geq 3$; the proof for $n=0,1,2$ can be established from the explicit formulas for $S_{m}, P_{m}, f_{m}$ in $\S 8$. 
Lemma 10.2. If (10.2)-(10.4) hold for all $m<n$ and $j \geq 0$ then, for all $k \geq 0$,

$$
\begin{gathered}
\left\|\partial_{\theta}^{k} F_{n}^{i}\right\|_{L^{2}(B)} \leq C_{0} M A^{k-2} H^{n-3} \frac{(k+n) !}{(k+n)^{3} n !} \quad(i=1,2), \\
\left\|\partial_{\theta}^{k} F_{n}^{3}\right\|_{H^{1 / 2}(\partial B)}+\left\|\partial_{\theta}^{k} F_{n}^{4}\right\|_{H^{3 / 2}(\partial B)} \leq C_{0} M A^{k-2} H^{n-3} \frac{(k+n) !}{(k+n)^{3} n !}
\end{gathered}
$$

where $M$ is a constant independent of $A, H$ and $k, n$.

Suppose the lemma is true. Then we can apply Lemma 8.2 to conclude that (10.2)-(10.4) hold for $m=n, j=0$ with $H^{n-2}$ replaced by $C M H^{n-3}$. The same estimates hold for $j=1$, using Lemma 8.3. For general $j$ we use either Lemma 8.2 (if $j$ is even) or Lemma 8.3 (if $j$ is odd). Note that the term

$$
\frac{\gamma_{n-1}}{R_{0}^{2}}\left(\ell^{2}-1\right) \cos \ell \theta
$$

in (7.19) is replaced, for $j$ even, by

$$
\frac{\gamma_{n-1}}{R_{0}^{2}}\left(\ell^{2}-1\right) \ell^{j}(-1)^{j / 2} \cos \ell \theta
$$

but this does not affect the estimates; the same is true if $j$ is odd (with $\cos \ell \theta$ replaced by $(-1)^{\frac{j+1}{2}} \sin \ell \theta$ ). Thus, choosing $H>M C$, we conclude that in order to prove (10.2)-(10.4) it suffices to prove Lemma 10.2.

Proof of Lemma 10.2. We introduce auxiliary functions which will appear in the expressions for the $F_{n}^{i}$. First,

$$
\begin{gathered}
-\frac{1}{R_{0}+f}=-\frac{1}{R_{0}}+\sum_{n \geq 1} \frac{(-1)^{n} f^{n}}{R_{0}^{n}} \equiv \sum_{n \geq 0} G_{n}(\theta) \varepsilon^{n}, \\
\log \left(R_{0}+f\right)=\log R_{0}+\sum_{n \geq 1}(-1)^{n-1} \frac{f^{n}}{n R_{0}^{n}} \equiv \sum_{n \geq 0} L_{n}(\theta) \varepsilon^{n} .
\end{gathered}
$$

By Lemma 9.1 and the inductive assumption (10.3),

$$
\left\|D_{\theta}^{k} G_{m}\right\|_{H^{7 / 2}(\partial B)}+\left\|D_{\theta}^{k} L_{m}\right\|_{H^{7 / 2}(\partial B)} \leq K C_{0} \frac{(k+m) !}{(k+m)^{3} m !} A^{k-2} H^{m-2} .
$$

We have

$$
\begin{gathered}
\frac{f_{\theta}}{R_{0}+f}=\sum_{n \geq 1} L_{n}^{\prime}(\theta) \varepsilon^{n} \\
\left(\frac{f_{\theta}}{R_{0}+f}\right)^{2}=\sum_{n \geq 2}\left(\sum_{k=1}^{n-1} L_{n-k}^{\prime} L_{k}^{\prime}\right) \varepsilon^{n} \equiv \sum_{n \geq 2} D_{n}(\theta) \varepsilon^{n}
\end{gathered}
$$

and by (9.7) (with $m=2$ )

$$
\left\|D_{\theta}^{k} D_{m}\right\|_{H^{5 / 2}(\partial B)} \leq K C_{0} \frac{(k+m) !}{(k+m)^{3} m !} A^{k-2} H^{m-3} .
$$

Next

$$
\frac{f_{\theta \theta}}{R_{0}+f}=\left(\log \left(R_{0}+f\right)\right)^{\prime \prime}+\left(\frac{f_{\theta}}{R_{0}+f}\right)^{2}=\sum_{n \geq 1}\left(L_{n}^{\prime \prime}+D_{n}\right) \varepsilon^{n}
$$


Writing

$$
f^{2}=\sum_{n \geq 2} Q_{n}(\theta) \varepsilon^{n}
$$

we have (again by (9.7) with $m=2$ )

$$
\left\|D_{\theta}^{k} Q_{m}\right\|_{H^{7 / 2}(\partial B)} \leq K C_{0} \frac{(k+m) !}{(k+m)^{3} m !} A^{k-2} H^{m-3} .
$$

The estimate (10.9) holds for all $m<n$ and $k \geq 0$, while the estimates (10.12), (10.14) are valid for $m \leq n$ and $k \geq 0$.

We now write the $F_{n}^{i}$ explicitly from (7.3)-(7.7):

$$
\begin{aligned}
& F_{n}^{1}= \sum_{k=0}^{n-1}\left\{2 L_{n-k}^{\prime} \frac{1}{r} \frac{\partial^{2} S_{k}}{\partial \theta \partial r}-D_{n-k}\left(\frac{2}{r} \frac{\partial S_{k}}{\partial r}+\frac{\partial^{2} S_{k}}{\partial r^{2}}\right)\right. \\
&\left.0.15)+\left(L_{n-k}^{\prime \prime}+D_{n-k}-\frac{f_{n}^{\prime \prime}}{R_{0}} \delta_{k, 0}\right) \frac{1}{r} \frac{\partial S_{k}}{\partial r}+Q_{n-k} S_{k}+2 R_{0} f_{n-k}\left(S_{k}-S_{0} \delta_{k, 0}\right)\right\} .
\end{aligned}
$$

Similarly,

$$
\begin{aligned}
& F_{n}^{2}= \sum_{k=0}^{n-1}\left\{2 L_{n-k}^{\prime} \frac{1}{r} \frac{\partial^{2} P_{k}}{\partial \theta \partial r}-D_{n-k}\left(\frac{2}{r} \frac{\partial P_{k}}{\partial r}+\frac{\partial^{2} P_{k}}{\partial r^{2}}\right)\right. \\
&+\left(L_{n-k}^{\prime \prime}+D_{n-k}-\frac{f_{n}^{\prime \prime}}{R_{0}} \delta_{k, 0}\right) \frac{1}{r} \frac{\partial P_{k}}{\partial r}-Q_{n-k}\left(S_{k}-\tilde{\sigma} \delta_{k, 0}\right) \\
&\left.\quad-2 R_{0} f_{n-k}\left(S_{k}-S_{0} \delta_{k, 0}\right)\right\} .
\end{aligned}
$$

Finally, $F_{n}^{3}$ and $F_{n}^{4}$ can be written in the form

$$
\begin{aligned}
F_{n}^{3} & =\sum_{k=1}^{n-1} V_{n-k} \frac{\partial P_{k}}{\partial \theta} \\
F_{n}^{4} & =\sum_{k=1}^{n-2} \gamma_{k} U_{n-k}
\end{aligned}
$$

where

$$
\left\|D_{\theta}^{k} V_{m}\right\|_{H^{5 / 2}(\partial B)}+\left\|D_{\theta}^{k} U_{m}\right\|_{H^{3 / 2}(\partial B)} \leq K C_{0} \frac{(k+m) !}{(k+m)^{3} m !} A^{k-2} H^{m-2} .
$$

Indeed, this follows by applying Lemma 9.1 to estimate

$$
\begin{aligned}
& \left.\partial_{\varepsilon}^{k}\right|_{\varepsilon=0} \frac{f_{\theta}\left(R_{0}+f\right)}{\left(f_{\theta}^{2}+\left(R_{0}+f\right)^{2}\right)} \text { and } \\
& \left.\partial_{\varepsilon}^{k}\right|_{\varepsilon=0} \frac{\left(R_{0}+f\right)^{2}+2 f_{\theta}^{2}-\left(R_{0}+f\right) f_{\theta \theta}}{\left(\left(R_{0}+f\right)^{2}+f_{\theta}^{2}\right)^{3 / 2}}
\end{aligned}
$$

in $H^{5 / 2}(\partial B)$ and $H^{3 / 2}(\partial B)$ norms, respectively (recall that we have $H^{2+3 / 2}(\partial B)$ estimate on $f)$. 
Using (10.4) (with $m<n)$ and (10.19), we easily obtain

$$
\left\|\partial_{\theta}^{k} F_{n}^{4}\right\|_{H^{3 / 2}(\partial B)} \leq M C_{0} \frac{(k+n) !}{(k+n)^{3} n !} A^{k-2} H^{n-3}
$$

Next

$$
\begin{aligned}
& \left\|\partial_{\theta}^{k} F_{n}^{3}\right\|_{H^{1 / 2}(\partial B)} \leq \sum_{l=1}^{n-1}\left\|\partial_{\theta}^{k}\left(V_{n-l} \frac{\partial P_{l}}{\partial \theta}\right)\right\|_{H^{1 / 2}(\partial B)} \\
& \quad \leq \sum_{l=1}^{n-1} \sum_{m=0}^{k} \frac{k !}{m !(k-m) !}\left\|\partial_{\theta}^{k-m} V_{n-l} \cdot \partial_{\theta}^{m} \frac{\partial P_{l}}{\partial \theta}\right\|_{H^{1 / 2}(\partial B)} \\
& \quad \leq C \sum_{l=1}^{n-1} \sum_{m=0}^{k} \frac{k !}{m !(k-m) !}\left\|\partial_{\theta}^{k-m} V_{n-l}\right\|_{H^{5 / 2}(\partial B)}\left\|\partial_{\theta}^{m}\left(P_{l}-\frac{\gamma_{l}}{R_{0}}\right)\right\|_{H^{2}(B)}
\end{aligned}
$$

where we used (9.1) and the trace theorem. Using (10.19) and the inductive assumption we find that the right-hand side is bounded by

$$
\begin{aligned}
C \sum_{l=1}^{n-1} \sum_{m=0}^{k} \frac{k !}{m !(k-m) !} & K C_{0} \frac{(n-l+k-m) !}{(n-l+k-m)^{3}(n-l) !} A^{k-m-2} H^{n-l-2} \\
& \times C_{0} \frac{(l+m) !}{(l+m)^{3} l !} A^{m-2} H^{l-2} .
\end{aligned}
$$

Thus

$$
\left\|\partial_{\theta}^{k} F_{n}^{3}\right\|_{H^{1 / 2}(\partial B)} \leq K C C_{0}^{2} A^{k-2} H^{n-3} \frac{(k+n) !}{(k+n)^{3} n !} J
$$

where

$$
J=\sum_{l, m} \frac{k ! n !}{(k+n) !} \frac{(l+m) !}{m ! l !} \frac{(n-l+k-m) !}{(k-m) !(n-l) !} \frac{(k+n)^{3}}{(l+m)^{3}(k+n-(m+l))^{3}} .
$$

Using (9.2) we conclude that $J$ is bounded by a constant.

We next need to estimate $F_{n}^{2}$. We can write

$$
\begin{aligned}
F_{n}^{2} & =\sum_{l=1}^{n-1}\left\{2 L_{n-l}^{\prime} \frac{1}{r} \frac{\partial^{2} P_{l}}{\partial r \partial \theta}-D_{n-l}\left(\frac{2}{r} \frac{\partial P_{l}}{\partial r}+\frac{\partial^{2} P_{l}}{\partial r^{2}}\right)\right. \\
& \left.+\left(L_{n-l}^{\prime \prime}+D_{n-l}\right) \frac{1}{r} \frac{\partial P_{l}}{\partial r}-Q_{n-l} S_{l}-2 R_{0} f_{n-l} S_{l}\right\} \\
& -D_{n}\left(\frac{2}{r} \frac{\partial P_{0}}{\partial r}+\frac{\partial^{2} P_{0}}{\partial r^{2}}\right)+\left(E_{n}^{\prime \prime}+D_{n}\right) \frac{1}{r} \frac{\partial P_{0}}{\partial r}-Q_{n}\left(S_{0}-\tilde{\sigma}\right)
\end{aligned}
$$

where $E_{n} \equiv L_{n}-\frac{f_{n}}{R_{0}}$ involves only the $f_{m}$ with $m<n$. Using (9.1) and the trace 
theorem we get

$$
\begin{aligned}
\| \partial_{\theta}^{k} & F_{n}^{2} \|_{L^{2}(B)} \leq C \sum_{l=1}^{n-1} \sum_{m=0}^{k} \frac{k !}{m !(k-m) !}\left\{\left\|\partial_{\theta}^{k-m} L_{n-l}\right\|_{H^{2+3 / 2}(\partial B)}\left\|\frac{1}{r} \frac{\partial^{2}}{\partial r \partial \theta} \partial_{\theta}^{m} P_{l}\right\|_{L^{2}(B)}\right. \\
& +\left\|\partial_{\theta}^{k-m} D_{n-l}\right\|_{H^{1+3 / 2}}\left(\left\|\frac{1}{r} \frac{\partial}{\partial r} \partial_{\theta}^{m} P_{l}\right\|_{L^{2}(B)}+\left\|\frac{\partial^{2}}{\partial r^{2}} \partial_{\theta}^{m} P_{l}\right\|_{L^{2}(B)}\right) \\
& +\left(\left\|\partial_{\theta}^{k-m} L_{n-l}\right\|_{H^{2+3 / 2}(\partial B)}+\left\|\partial_{\theta}^{k-m} D_{n-l}\right\|_{H^{1+3 / 2}}\right)\left\|\frac{1}{r} \frac{\partial}{\partial r} \partial_{\theta}^{m} P_{l}\right\|_{L^{2}(B)} \\
& \left.+\left\|\partial_{\theta}^{k-m} Q_{n-l}\right\|_{H^{2+2 / 3}(\partial B)}\left\|\partial_{\theta}^{m} S_{l}\right\|_{L^{2}(B)}+\left\|\partial_{\theta}^{k-m} f_{n-l}\right\|_{H^{2+2 / 3}(\partial B)}\left\|\partial_{\theta}^{m} S_{l}\right\|_{L^{2}(B)}\right\} \\
& +C\left\|\partial_{\theta}^{k} D_{n}\right\|_{H^{1+3 / 2}(\partial B)}\left(\left\|\frac{1}{r} \frac{\partial P_{0}}{\partial r}\right\|_{L^{2}(B)}+\left\|\frac{\partial^{2} P_{0}}{\partial r^{2}}\right\|_{L^{2}(B)}\right) \\
& +C\left(\left\|\partial_{\theta}^{k} E_{n}\right\|_{H^{2+3 / 2}(\partial B)}+\left\|\partial_{\theta}^{k} D_{n}\right\|_{H^{1+3 / 2}(\partial B)}\right)\left\|\frac{1}{r} \frac{\partial P_{0}}{\partial r}\right\|_{L^{2}(B)} \\
& +C\left\|\partial_{\theta}^{k} Q_{n}\right\|_{H^{2+3 / 2}(\partial B)}\left(\left\|S_{0}\right\|_{L^{2}(B)}+1\right) .
\end{aligned}
$$

By the inductive assumption and (10.9), (10.12) (10.14) we find that the sum of the above terms is bounded by

$$
\begin{aligned}
C \sum_{\ell=1}^{n-1} & \sum_{m=0}^{k} \frac{k !}{m !(k-m) !} K C_{0} \frac{(n-l+k-m) !}{(n-l+k-m)^{3}(n-l) !} C_{0} \frac{(m+l) !}{(m+l)^{3} l !} \\
& \times A^{k-m-2} H^{n-l-2} A^{m-2} H^{l-2}+C K C_{0}^{2} \frac{(k+n) !}{(k+n)^{3} n !} A^{k-2} H^{n-3} \\
& \leq C K C_{0}^{2} S A^{k-2} H^{n-3} \frac{(k+n) !}{(k+n)^{3} n !} .
\end{aligned}
$$

Similarly, one can estimate $\left\|\partial_{\theta}^{k} F_{n}^{1}\right\|_{L^{2}(B)}$ and together with the above estimates on $F_{n}^{3}, F_{n}^{4}$, the proof of Lemma 10.2 is complete.

\section{Convergence (Continued)}

From the estimates of Lemma 10.2 we already conclude that $f(\theta, \varepsilon)$ is analytic in $(\theta, \varepsilon)$ and $\widehat{\sigma}(r, \theta, \varepsilon), \widehat{p}(r, \theta, \varepsilon)$ are analytic in $(\theta, \varepsilon)$ for $0 \leq \theta \leq 2 \pi,|\varepsilon| \leq \varepsilon_{0}$. In this section we want to extend the estimates to all $r$-derivatives so as to conclude the joint analyticity of $\widehat{\sigma}, \widehat{p}$ in $r, \theta, \varepsilon$ up to $r=1$ (and actually to $r \leq 1+\delta_{0}$ for some small positive $\delta_{0}$ ).

We shall prove the following:

Lemma 11.1. For all $k \geq 0, n \geq 0, h>0$

$$
\begin{aligned}
\left\|r^{h} \partial_{r}^{h} \partial_{\theta}^{k} S_{n}\right\|_{L^{2}(B)} & +\left\|r^{h} \frac{1}{r} \frac{\partial}{\partial r} \partial_{r}^{h} \partial_{\theta}^{k} S_{n}\right\|_{L^{2}(B)}+\left\|r^{h} \frac{1}{r^{2}} \frac{\partial}{\partial \theta} \partial_{r}^{h} \partial_{\theta}^{k} S_{n}\right\|_{L^{2}(B)} \\
& +\left\|r^{h} \frac{1}{r^{2}} \frac{\partial^{2}}{\partial \theta^{2}} \partial_{r}^{h} \partial_{\theta}^{k} S_{n}\right\|_{L^{2}(B)}+\left\|r^{h} \frac{1}{r} \frac{\partial^{2}}{\partial r \partial \theta} \partial_{r}^{h} \partial_{\theta}^{k} S_{n}\right\|_{L^{2}(B)} \\
& +\left\|r^{h} \frac{\partial^{2}}{\partial r^{2}} \partial_{r}^{h} \partial_{\theta}^{k} S_{n}\right\|_{L^{2}(B)} \leq C_{0} \frac{(k+h+n) !}{(k+h+n)^{3} n !} B^{h} A^{k-2} H^{n-2} ;
\end{aligned}
$$

the same estimates hold for $P_{n}$. 
Proof. From (7.15) we have

$$
\begin{aligned}
\frac{\partial^{2}}{\partial r^{2}} \partial_{r}^{j} \partial_{\theta}^{k} S_{n} & =\partial_{r}^{j} \partial_{\theta}^{k} F_{n}^{1}-\partial_{r}^{j}\left(\frac{1}{r} \partial_{\theta}^{k} S_{n}\right)-\partial_{r}^{j}\left(\frac{1}{r^{2}} \frac{\partial^{2}}{\partial \theta^{2}} \partial_{\theta}^{k} S_{n}\right) \\
& +\left(\partial_{\theta}^{k} \wedge_{n, \theta \theta}\right) \frac{1}{R_{0}} \partial_{r}^{j}\left(\frac{1}{r} \partial_{r} S_{0}\right)+R_{0}^{2} \partial_{r}^{j} \partial_{\theta}^{k} S_{n}+2\left(\partial_{\theta}^{k} f_{n}\right) R_{0} \partial_{r}^{j} S_{0}
\end{aligned}
$$

We assume inductively that (11.1) and its analog for $P_{n}$ hold for all $h$ smaller than $j$ and proceed to prove it for $j$; for $j=0$ this follows from (10.2), (10.3). We shall consider in detail the term

$$
J_{1}=r^{j} \partial_{r}^{j} \frac{1}{r^{2}} \frac{\partial^{2}}{\partial \theta^{2}} \partial_{\theta}^{k} S_{n}
$$

and a typical term from $\partial_{r}^{j} \partial_{\theta}^{k} F_{n}^{1}$ (cf. (10.15)), namely,

$$
J_{2}=r^{j} \partial_{r}^{j} \partial_{\theta}^{k} \sum_{t=1}^{n-1} L_{n-t}^{\prime} \frac{1}{r} \frac{\partial^{2}}{\partial \theta \partial r} S_{t}
$$

all the other terms on the right-hand side of (11.2) can be estimated in a similar way.

We can write

$$
\begin{aligned}
J_{1}= & r^{j} \sum_{s=0}^{j}(-1)^{j-s} r^{-2-(j-s)}(j-s+1) ! \frac{j !}{s !(j-s) !} \partial_{\theta}^{2} \partial_{\theta}^{k} \partial_{r}^{s} S_{n} \\
= & \sum_{s=1}^{j} \frac{j !}{s !(j-s) !}(j-s+1) !(-1)^{j-s} r^{s-1} \frac{1}{r} \partial_{r} \partial_{\theta} \partial_{\theta}^{k+1} \partial_{r}^{s-1} S_{n} \\
& +(-1)^{j}(j+1) ! \frac{1}{r^{2}} \partial_{\theta}^{2} \partial_{\theta}^{k} S_{n} .
\end{aligned}
$$

The last term is bounded in $L^{2}(B)$ by

$$
\begin{aligned}
& C_{0} A^{k-2} H^{n-2} \frac{(k+n) !(j+1) !}{(k+n)^{3} n !} \\
& \quad \leq C_{0} A^{k-2} H^{n-2} B^{j} \frac{(k+j+n) !}{(k+j+n)^{3} n !}\left[\frac{(k+n) !(j+1) !}{(k+n+j) !} \frac{(k+n+j)^{3}}{(k+n)^{3}} B^{-j}\right] \\
& \quad \leq C_{0} A^{k-2} H^{n-2} B^{j} \frac{(k+j+n) !}{(k+j+n)^{3} n !}\left[(j+1)^{4} B^{-j}\right],
\end{aligned}
$$

whereas the $L^{2}(B)$ norm of the sum is bounded by

$$
\begin{aligned}
\sum_{s=1}^{j} & \frac{j !}{s !(j-s) !}(j-s+1) ! C_{0} A^{k-1} B^{s-1} H^{n-2} \frac{(k+s+n) !}{(k+s+n)^{3} n !} \\
\leq & C_{0} H^{n-2} B^{j} A^{k-2} \frac{(k+n+j) !}{(k+n+j)^{3} n !} \frac{A}{B} \\
& \times \sum_{s=1}^{j} B^{-(j-s)} \frac{(k+s+n) !}{(k+n+j) !} \frac{(k+n+j)^{3}}{(k+n+s)^{3}}(j-s+1) \frac{j !}{s !}
\end{aligned}
$$

By (9.2)

$$
\frac{(k+s+n) !}{(k+n+j) !} \frac{j !}{s !}=\left(\begin{array}{l}
j \\
s
\end{array}\right) /\left(\begin{array}{l}
k+n+j \\
k+n+s
\end{array}\right) \leq 1
$$


so that the expression in the last sum is less than

$$
\sum_{s=1}^{j}(1+j-s) \frac{(k+n+j)^{3}}{(k+n+s)^{3}} B^{-(j-s)} \leq C \sum_{s=1}^{j}(1+(j-s))^{4} B^{-(j-s)} .
$$

Taking $B$ large enough we get the bound

$$
\left\|J_{1}\right\|_{L^{2}(B)} \leq C_{0} H^{n-2} B^{j} A^{k-2} \frac{(k+j+n) !}{(k+j+n)^{3} n !} .
$$

To estimate $J_{2}$ we write

$$
\begin{aligned}
J_{2}= & \sum_{t=1}^{n-1} \sum_{p=0}^{k} \sum_{s=0}^{j} \frac{k !}{p !(k-p) !} \frac{j !}{s !(j-s) !} \partial_{\theta}^{k-p} \partial_{\theta} L_{n-t}(-1)^{j-s} r^{j} r^{-(j-s)}(j-s) ! \\
& \times \frac{1}{r} \frac{\partial^{2}}{\partial r \partial \theta} \partial_{\theta}^{p} \partial_{r}^{s} S_{t} \\
= & \sum_{t=1}^{n-1} \sum_{p=0}^{k} \sum_{s=0}^{j-1} \frac{k !}{p !(k-p) !} \frac{j !}{s !(j-s) !} \partial_{\theta}^{k-p} \partial_{\theta} L_{n-t}(-1)^{j-s} r^{s}(j-s) ! \frac{1}{r} \frac{\partial^{2}}{\partial r \partial \theta} \partial_{\theta}^{p} \partial_{r}^{s} S_{t} \\
& \quad+\sum_{t=1}^{n-1} \sum_{p=0}^{k} \frac{k !}{p !(k-p) !} \partial_{\theta}^{k-p} \partial_{\theta} L_{n-t} r^{j-1} \frac{\partial^{2}}{\partial r^{2}} \partial_{\theta}^{p+1} \partial_{r}^{j-1} S_{t} .
\end{aligned}
$$

The $L^{2}(B)$ norm of the last sum can be estimated by

$$
\begin{aligned}
& \sum_{t, p} \frac{k !}{p !(k-p) !} K C_{0} \frac{(k-p+n-t) !}{(k-p+n-t)^{3}(n-t) !} A^{k-p-2} H^{n-t-2} \\
& \quad \times C_{0} \frac{(p+j+t) !}{(p+j+t)^{3} t !} B^{j-1} A^{p-1} H^{t-2} \\
& \leq K C_{0}^{2} A^{k-2} H^{n-3} B^{j} \frac{(k+n+j) !}{(k+n+j)^{3} n !} \frac{A}{B} \\
& \quad \times \sum_{t, p} \frac{(k+n+j)^{3}}{((k+n+j)-(p+j+t))^{3}(p+j+t)^{3}} \frac{\left(\begin{array}{c}
n \\
t
\end{array}\right)}{\left(\begin{array}{c}
k+n+j \\
p+t+j
\end{array}\right)}
\end{aligned}
$$

and, since $\left(\begin{array}{l}n \\ t\end{array}\right) \leq\left(\begin{array}{c}k+n+j \\ p+t+j\end{array}\right)($ by $(9.2))$,

$$
\begin{gathered}
\sum_{t, p} \frac{(k+n+j)^{3}}{((k+n+j)-(p+j+t))^{3}(p+j+t)^{3}} \frac{\left(\begin{array}{l}
n \\
t
\end{array}\right)}{\left(\begin{array}{l}
k+n+j \\
p+t+j
\end{array}\right)} \\
\leq \sum_{t, p} \frac{(k+n+j)^{3}}{((k+n+j)-(p+j+t))^{3}(p+j+t)^{3}}<\infty
\end{gathered}
$$


Similary, the $L^{2}(B)$ norm of the first sum in the expression for $J_{2}$ is bounded by

$$
\begin{aligned}
& \sum_{t, p, s} \frac{k !}{p !(k-p) !} \frac{j !}{s !(j-s) !}(j-s) ! K C_{0} \frac{(k-p+n-t) !}{(k-p+n-t)^{3}(n-t) !} \times A^{k-p-2} H^{n-t-2} \\
& \quad \times C_{0} \frac{(p+s+t) !}{(p+s+t)^{3} t !} B^{s} A^{p-2} H^{t-2} \\
& \leq K C_{0}^{2} A^{k-2} H^{n-3} B^{j} \frac{(k+n+j) !}{(k+n+j)^{3} n !} \sum_{s=0}^{j-1} \sum_{t, p} B^{-(j-s)} \\
& \quad \times\left[\frac{(k-p+n-t) !(p+t+s) !}{(k+n+s) !} \frac{(k+n) !}{(k+n+j) !}(j-s) !\left(\begin{array}{l}
j \\
s
\end{array}\right)\left(\begin{array}{c}
n \\
t
\end{array}\right)\left(\begin{array}{l}
k \\
p
\end{array}\right)\right] \\
& \quad \times \frac{(k+n+j)^{3}}{(k+n-p-t)^{3}(p+s+t)^{3}}
\end{aligned}
$$

and, by (9.2), the expression in brackets is bounded by

$$
\begin{aligned}
& \frac{(k-p+n-t) !(p+t+s) !}{(k+n+s) !} \frac{(k+n+s) !}{(k+n+j) !}\left(\begin{array}{c}
n+k+j \\
p+t+s
\end{array}\right)(j-s) ! \\
& =\frac{(k+n-(p+t)) !(j-s) !}{(n+k+j-(p+t+s)) !} \leq 1 /\left(\begin{array}{c}
n+k+j-(t+p+s) \\
j-s
\end{array}\right) \leq 1 .
\end{aligned}
$$

Consequently, after choosing $B$ large enough, we get the same bound as for $J_{1}$.

As mentioned above, all other terms can be estimated in a similar way.

From Theorem 10.1 and Lemma 11.1 we deduce the convergence of the series (7.9)-(7.12) and the analyticity of $\widehat{\sigma}(r, \theta, \varepsilon), \widehat{p}(r, \theta, \varepsilon)$ in $(r, \theta, \varepsilon)$ in the region $0<$ $r \leq 1+\delta_{0}, 0 \leq \theta \leq 2 \pi,|\varepsilon| \leq \varepsilon_{0}$ for some $\delta_{0}>0, \varepsilon_{0}>0$.

From the transformation (7.1), (7.2) it follows that $\sigma(x, \varepsilon)$ and $p(x, \varepsilon)$ are analytic functions jointly in $x=\left(x_{1}, x_{2}\right)$ and $\varepsilon$ for $\eta<|x| \leq R_{0}+\delta_{0},|\varepsilon| \leq \varepsilon_{0}$ where $\eta$ is any positive number. Since $\sigma(x, \varepsilon)$ is bounded in a neighborhood of $x=0$, the singularity at $x=0$ is removable and we can represent $\sigma$ in terms of the fundamental solution $G(x)=\frac{1}{2 \pi} K_{0}(|x|)$ where $K_{0}(z)$ is the modified Bessel function of the third kind and order 0 ,

$$
\sigma(x, \varepsilon)=-\int_{|y|=\eta_{0}} \frac{\partial G}{\partial n}(x-y) \sigma(y, \varepsilon) d S_{y}+\int_{|y|=\eta_{0}} G(x-y) \frac{\partial}{\partial n} \sigma(y, \varepsilon) d S_{y}
$$

for $|x|<\eta_{0}$ where $\eta_{0}>\eta$. From this representation we deduce the analyticity of $\sigma(x, \varepsilon)$ in $(x, \varepsilon)$ for $|x| \leq \eta,|\varepsilon| \leq \varepsilon_{0}$.

Finally, since the function $w=p+\mu \sigma+\mu \frac{\left(x^{2}+y^{2}\right)}{4} \tilde{\sigma}$ is harmonic, a similar argument to the one above (now with the fundamental solution $H(x)=\frac{1}{2 \pi} \log \frac{1}{|x|}$ ) implies the analyticity of $w$ in a neighborhood of the origin. The corresponding analyticity for $p$ can then be derived from the representation $p=w-\mu \sigma-\mu\left(x^{2}+y^{2}\right) \frac{\tilde{\sigma}}{4}$.

We summarize:

Theorem 11.2. For any $R_{0}>0$, and $f_{1}(\theta)=\cos \ell \theta(\ell \geq 2)$, there exists a family of solutions, even in $\theta,(\sigma, p, f, \gamma)$ of (1.1)-(1.5) of the form (1.7), for all $|\varepsilon| \leq \varepsilon_{0}$, where $\varepsilon_{0}$ is a positive number; the series are convergent for $|\varepsilon| \leq \varepsilon_{0}$ and $|x| \leq R_{0}+\delta_{0}$ (for some positive $\delta_{0}$ ) and are analytic functions in $(x, y, \varepsilon)$ for $\sqrt{x^{2}+y^{2}} \leq R_{0}+$ 
$\delta_{0},|\varepsilon| \leq \varepsilon_{0}$. For each $\varepsilon$, the solution is unique under the orthogonality conditions:

$$
\begin{aligned}
& \int_{0}^{2 \pi} f_{j}(\theta) \cos (m \theta) d \theta=0 \quad \text { for } m=1, \ell \text { and } j \geq 2, \\
& \int_{0}^{2 \pi} \sigma \cos \theta d \theta=\int_{0}^{2 \pi} p \cos \theta d \theta=0 \quad \forall 0<r \leq R_{0} .
\end{aligned}
$$

\section{A further application of THE METHOD}

In $\S 6$ we motivated our approach to the proof of convergence (given in $\S \S 7-11$ ) by the example (6.4), (6.5). We now show that this method actually works for this example. For simplicity we assume (as in [4]) that

$$
f(x) \text { and } H(x) \text { are } 2 \pi \text {-periodic. }
$$

We seek a solution of (6.18), (6.19) which is $2 \pi$-periodic in $x$ and remains bounded as $z \rightarrow \infty$.

Equation (6.21) can be written in more detail in the form

$$
\Delta v_{n}=-\left(f^{\prime}\right)^{2} \partial_{z}^{2} v_{n-2}+2 f^{\prime} \partial_{x} \partial_{z} v_{n-1}+f^{\prime \prime} \partial_{z} v_{n-1} \equiv F_{n} \quad \text { if } \quad n \geq 0 .
$$

Equation (12.2) is analogous to the system (7.15)-(7.19).

We need to show that (12.2) with boundary condition $v_{n}(x, 0)=0 \quad(n \geq 1)$ has a unique $2 \pi$-periodic (in $x$ ) solution, and to derive $H^{2}$ bounds on $v_{n}$. For this purpose we write

$$
f(x)=\sum c_{q} e^{i q x}
$$

and expand formally

$$
v_{n}(z)=\sum_{r} d_{n, r}(z) e^{i r x}
$$

Substituting these expressions into (12.2) we get, after equating the coefficients of $e^{i r x}$,

$$
d_{n, r}^{\prime \prime}-r^{2} d_{n r}=F_{n r}
$$

where $F_{n r}$ depends only on the $d_{m, s}$ for $m<n$. From this relation we deduce uniqueness.

To prove existence and analyticity, we establish a result similar to Lemma 8.1, with weighted norms

$$
\|k\|_{\lambda}^{2}=\frac{1}{2 \pi} \int_{0}^{\infty}\left(\int_{0}^{2 \pi}|k(x, z)|^{2} d x\right) e^{\lambda z} d z .
$$

Lemma 12.1. Let $g(x, z)$ be a function $2 \pi$-periodic in $x$ with $\|g\|_{\lambda}<\infty \quad(\lambda>0)$ and consider the problem

$$
\begin{gathered}
\Delta w=g \quad \text { in } \quad z>0, \\
w=0 \quad \text { on } \quad z=0 .
\end{gathered}
$$

Then there exists a unique solution $w$ which is $2 \pi$-periodic in $x$ such that

$$
\left\|\partial_{z} w\right\|_{\lambda}+\left\|\partial_{z} \partial_{x} w\right\|_{\lambda}+\left\|\partial_{z}^{2} w\right\|_{\lambda} \leq C\|g\|_{\lambda} .
$$


Proof. We expand

$$
\begin{aligned}
& g(x, z)=\sum_{r} g_{r}(z) e^{i r x}, \\
& w(x, z)=\sum_{r} w_{r}(z) e^{i r x},
\end{aligned}
$$

so that $(12.3),(12.4)$ reduce to

$$
w_{r}^{\prime \prime}-r^{2} w_{r}=g_{r}(z), \quad w_{r}(0)=0
$$

and

$$
\int_{0}^{\infty}\left|g_{r}(z)\right|^{2} e^{\lambda z} d z<\infty
$$

The only solution to this problem is given by

$$
\begin{gathered}
w_{r}(z)=-\frac{e^{|r| z}}{2|r|} \int_{z}^{\infty} e^{-|r| s} g_{r}(s) d s+\frac{e^{-|r| z}}{2|r|} \int_{0}^{z} g_{r}(s) e^{|r| s} d s \\
+\int_{0}^{\infty} g_{r}(s) e^{-|r| s} \frac{e^{-|r| z}}{2|r|} d s+c_{r}\left(e^{|r| z}-e^{-|r| z}\right) \quad \text { if } \quad r \geq 1 \\
w_{0}(z)=-\int_{0}^{z} s g(s) d s-\int_{z}^{\infty} z g(s) d s+c_{0} z
\end{gathered}
$$

where the $c_{r}$ are constants. The assumption of finite norm (in particular, $\left.\left\|\partial_{z}\left(w_{r}(z) e^{i r x}\right)\right\|_{\lambda}<\infty\right)$ implies that $c_{r}=0$ if $r \geq 0$. Let us now prove that

$$
\left(\int_{0}^{\infty}\left|w_{r}^{\prime}(z)\right|^{2} e^{\lambda z} d z\right)^{1 / 2} \leq C\left(\int_{0}^{\infty}\left|g_{r}(s)\right|^{2} e^{\lambda s} d s\right)^{1 / 2} .
$$

From (12.6) we get

$$
\begin{gathered}
w_{r}^{\prime}(z)=-\frac{1}{2} e^{|r| z} \int_{z}^{\infty} e^{-|r| s} g_{r}(s) d s-\frac{1}{2} e^{-|r| z} \int_{0}^{z} g_{r}(s) e^{|r| s} d s \\
-\frac{1}{2} \int_{0}^{\infty} g_{r}(s) e^{-|r| s} e^{-|r| z} d s, \quad r \geq 1 \\
w_{0}^{\prime}(z)=-\int_{z}^{\infty} g_{0}(s) d s .
\end{gathered}
$$

Consider the first term in $w_{r}^{\prime}(z), r \geq 1$ :

$$
\begin{aligned}
& \left(\int_{0}^{\infty}\left|e^{|r| z} \int_{z}^{\infty} e^{-|r| s} g_{r}(s) d s\right|^{2} e^{\lambda z} d z\right)^{1 / 2} \\
& \quad=\left(\int_{0}^{\infty}\left|\int_{0}^{\infty} e^{-|r| u} g_{r}(u+z) d u\right|^{2} e^{\lambda z} d z\right)^{1 / 2} \\
& \quad \leq \int_{0}^{\infty} e^{-|r| u}\left(\int_{0}^{\infty} g_{r}(u+z)^{2} e^{\lambda z} d z\right)^{1 / 2} d u \quad \text { (by Minkowski's inequality) } \\
& \quad=\int_{0}^{\infty} e^{-|r| u}\left(\int_{u}^{\infty} g_{r}(s)^{2} e^{\lambda(s-u)} d s\right)^{1 / 2} d u \leq \frac{1}{|r|+\lambda / 2}\left(\int_{0}^{\infty} g_{r}(s)^{2} e^{\lambda s} d s\right)^{1 / 2} .
\end{aligned}
$$


Similarly, one can estimate the other terms in $w_{r}^{\prime}$. To deal with $w_{0}^{\prime}$ we estimate

$$
\begin{aligned}
& \left(\int_{0}^{\infty}\left|w_{0}^{\prime}(z)\right|^{2} e^{\lambda z} d z\right)^{1 / 2}=\left(\int_{0}^{\infty}\left(\int_{z}^{\infty} g_{0}(s) d s\right)^{2} e^{\lambda z} d z\right)^{1 / 2} \\
& \quad=\left(\int_{0}^{\infty}\left(\int_{0}^{\infty} g_{0}(u+z) d u\right)^{2} e^{\lambda z} d z\right)^{1 / 2} \\
& \quad \leq \int_{0}^{\infty} d u\left(\int_{0}^{\infty} g_{0}(u+z)^{2} e^{\lambda z} d z\right)^{1 / 2}=\int_{0}^{\infty} d u\left(\int_{u}^{\infty} g_{0}(s)^{2} e^{\lambda z} d s\right)^{1 / 2} e^{-\lambda u / 2} \\
& \quad \leq \frac{2}{\lambda}\left(\int_{0}^{\infty} g_{0}(s)^{2} e^{\lambda s} d s\right)^{1 / 2} .
\end{aligned}
$$

Thus, we conclude that

$$
\left\|\partial_{z} w\right\|_{\lambda} \leq C\|g\|_{\lambda}
$$

The other norms

$$
\left\|\partial_{z} \partial_{x} w\right\|_{\lambda}, \quad\left\|\partial_{z}^{2} w\right\|_{\lambda}
$$

can be estimated in the same way.

Having proved Lemma 12.1 we now follow the procedure of $\S \S 10,11$ and establish the analyticity of $v(x, z, \varepsilon)$. Then, by a change of variables, we conclude the existence of $2 \pi$-periodic (in $x)$ solution $u(x, y, \varepsilon)$ of $(6.4),(6.5)$ which is analytic in $(x, y, \varepsilon)$ for $-\infty<x<\infty,-\delta_{0} \leq y<\infty,|\varepsilon| \leq \varepsilon_{0}$, where $\delta_{0}, \varepsilon_{0}$ are small positive constants.

\section{ACKNOWLEDGMENT}

The first author is partially supported by the National Science Foundation Grant DMS 9970522. The second author is partially supported by AFOSR through contract number F49620-99-1-0193 and by NSF through grants DMS-9622555 and DMS-9971379. The authors with to extend their thanks to Bei Hu and Juan J. L. Velazquez for useful discussions during the preparation of the manuscript.

\section{ACKNOWLEDGMENT AND DISCLAIMER}

Effort sponsored by the Air Force Office of Scientific Research, Air Force Materials Command, USAF, under grant no. F49620-99-1-0193. The US Government is authorized to reproduce and distribute reprints for governmental purposes notwithstanding any copyright notation thereon. The views and conclusions contained herein are those of the authors and should not be interpreted as necessarily representing the official policies or endorsements, either expressed or implied, of the Air Force Office of Scientific Research or the US Government.

\section{REFERENCES}

1. J.A. Adam, General aspects of modeling tumor growth and immune response, in A Survey of Models for Tumor-Immune System Dynamics, J.A. Adam and N. Bellomo, eds., Birkhäuser, Boston (1996), 15-87.

2. R.A. Adams, Sobolev Spaces, Academic Press, New York (1975). MR 56:9247]

3. O. Bruno and P. Laurence, Existence of three-dimensional toroidal MHD equilibria with nonconstant pressure, Comm. Pure Appl. Math., 49 (1996), 717-764. MR 97g:35137

4. O. Bruno and F. Reitich, Solution of the boundary value problem for the Helmholz equation via variation of the boundary into the complex domain, Proc. Roy. Soc. Edinburgh, Sec. A 122 (1992), 317-340. MR 94e:35131 
5. H.M. Byrne, The importance of intercellular adhesion in the development of carcinomas, IMA J. Math. Appl. Med. and Biol., 14 (1997), 305-323.

6. H.M. Byrne, The effect of time delays on the dynamics of avascular tumor growth, Math. Biosciences, 144 (1997), 83-117. MR 98h:92014.

7. H.M. Byrne and M.A.J. Chaplain, Growth of necrotic tumors in the presence and absence of inhibitors, Math. Biosciences, 135 (1996), 187-216.

8. M.A.J. Chaplain, The development of a spatial pattern in a model for cancer growth, in Experimental and Theoretical Advances in Biological Pattern Formation, H.G. Othmer, P.K. Maini and J.D. Murray eds., Plenum Press (1993), 45-59. MR 22:9739

9. A. Friedman, On the regularity of solutions of nonlinear elliptic and parabolic systems of partial differential equations, J. Math. Mech., 7 (1958), 43-60.

10. A. Friedman and F. Reitich, Analysis of a mathematical model for the growth of tumors, J. Math. Biol., 38 (1999), 262-284. CMP 99:11

11. A. Friedman and F. Reitich, Nonlinear stability of a quasi-static Stefan problem with surface tension: a continuation approach, to appear.

12. H.P. Greenspan, On the growth and stability of cell cultures and solid tumors, Theor. Biol., 56 (1976), 229-242. MR 55:2183

13. D.L.S. McElwain and L.E. Morris, Apoptosis as a volume loss mechanism in mathematical models of solid tumor growth, Math. Biosciences, 39 (1978), 147-157.

14. D.H Sattinger, Group-Theoretic Methods in Bifurcation Theory, Lecture Notes in Mathematics, 762, Springer-Verlag, Berlin (1979). MR 81e:58022

15. J. Smoller, Shock Waves and Reaction-Diffusion Equations, Springer-Verlag, New York (1983). MR 84d:35002

16. G.N. Watson, A Treatise on the Theory of Bessel Functions, Second Edition, Cambridge University Press (1944). MR 6:64a

School of Mathematics, University of Minnesota, Minneapolis, Minnesota 55455

E-mail address: friedman@math.umn.edu

School of Mathematics, University of Minnesota, Minneapolis, Minnesota 55455

E-mail address: reitich@math.umn.edu 